\title{
Eigenvalue Attraction
}

\author{
Ramis Movassagh ${ }^{1, \text { ⿵ }}$ \\ ${ }^{1}$ Department of Mathematics, IBM T.J. Watson Research Center, Yorktown Heights, NY, 10598
}

(Dated: February 26, 2016)

\begin{abstract}
We prove that the complex conjugate (c.c.) eigenvalues of a smoothly varying real matrix attract (Eq. 15. We offer a dynamical perspective on the motion and interaction of the eigenvalues in the complex plane, derive their governing equations and discuss applications. C.c. pairs closest to the real axis, or those that are ill-conditioned, attract most strongly and can collide to become exactly real. As an application we consider random perturbations of a fixed matrix $M$. If $M$ is Normal, the total expected force on any eigenvalue is shown to be only the attraction of its c.c. (Eq. 24) and when $M$ is circulant the strength of interaction can be related to the power spectrum of white noise. We extend this by calculating the expected force (Eq. 41 for real stochastic processes with zero-mean and independent intervals. To quantify the dominance of the c.c. attraction, we calculate the variance of other forces. We apply the results to the Hatano-Nelson model and provide other numerical illustrations. It is our hope that the simple dynamical perspective herein might help better understanding of the aggregation and low density of the eigenvalues of real random matrices on and near the real line respectively. In the appendix we provide a Matlab code for plotting the trajectories of the eigenvalues.
\end{abstract}

\section{Contents}

I. Background, illustration and summary of main results

A. Background

B. An illustration

C. Summary of the main results

II. Eigenvalues as a many-body system

A. General dynamics of eigenvalues

B. Definition and Proof of Eigenvalue Attraction

III. Applications of eigenvalue attraction

A. Smooth interpolation between fixed matrices

B. Random perturbations of a fixed matrix 9

1. First variation $\quad 10$

2. Second variation

C. Normal and circulant matrices

D. Hatano-Nelson model

E. Further illustrations $\quad[18$

IV. Special case of stochastic dynamics of the eigenvalues $\quad[18$

A. A discrete stochastic process $\quad[18$

B. Smoothened discrete stochastic process $\quad 19$

V. Further discussions and open problems

A. Does the low density of eigenvalues near the real line result from repulsion?

B. Further opportunities for future work

Acknowledgements

References 24

VI. Appendix: Matlab code $\quad[26$ 


\section{BACKGROUND, ILLUSTRATION AND SUMMARY OF MAIN RESULTS}

\section{A. Background}

Much work has been devoted to the understanding of the behavior of eigenvalues in the presence of randomness. The folklore of random matrix analysis, especially in the case of Hermitian matrices, suggests that the eigenvalues of a perturbed matrix repel. This has been pointed out previously by various authors [1, 2] and is well known in quantum physics [3. p. 304-305]. More recently, in agreement with the universality conjectures, the level repulsion was proved for the eigenvalues of a Wigner matrix [4].

The stochastic dynamics of the eigenvalues of Hermitian matrices have been vigorously studied in the past [5, recommended]. Most celebrated is Dyson's Brownian motion, which proves that the eigenvalues of a Hermitian matrix undergoing a Wiener process perform a Brownian motion [6].

In physics, one mainly studies Hermitian matrices and operators as their eigenvalues correspond to observable quantities, which need to be real. However, in recent years, non-Hermitian models have gained much attention in the context of pinning of vortices in type II superconductors initiated by Hatano and Nelson [7] and followed up in works on the nature of localized states and eigenvalue distributions [8-11]. Non-Hermitian models also come up in fluid mechanics [1, Ref. therein], transport phenomena in photonics [12] and biophysical phenomena [13].

In the Hatano-Nelson model, the eigenvalue distribution gives rise to "wings" of real eigenvalues when the perturbation is sufficiently strong (see for example [1, Section 36] and citations therein as well as Figs. 1 and 6). The wings result from the motion of complex eigenvalues that move in response to the perturbation and ultimately sit on the real axis. Goldsheid et al derived an equation for the shape of "the winged" spectrum [14]. According to [7], these eigenvalues correspond to localized eigenstates.

In investigating the (de)localization of the eigenstates, Feinberg and Zee [10], argued that imaginary eigenvalues near the real axis can attract when perturbed by a Hermitian matrix by providing a $2 \times 2$ example of an imaginary diagonal matrix perturbed by a $2 \times 2$ Hermitian matrix with zero diagonal entries. Later, Bloch et al [15] considered antisymmetric perturbations of real symmetric matrices in the context of two-color quantum chromodynamics and provided examples that a Hermitian matrix perturbed by a real antisymmetric perturbation can give rise to attraction of eigenvalues. To our knowledge, attraction of the eigenvalues and their eventual aggregation on the real line, in a general setting, has not been proved.

In this paper, we prove a simple theorem, which under very mild set of assumptions shows that the complex conjugate eigenvalues of a smoothly varying real matrix $M(t)$ attract (i.e., pull on each other). We then consider probabilistic settings where the underlying evolution of the matrix is random and prove statistical dynamical properties of any given eigenvalue. We extend the results to real stochastic processes, which naturally leads to a conjecture on the cause of aggregation and low density of the eigenvalues of real random matrices on and near the real line. A main emphasis of this work is a many-body dynamical perspective on the interaction and motion of the eigenvalues in the complex plane. Throughout, we illustrate the theory with examples and numerical results.

\section{B. An illustration}

Remark 1. Explanation of the figures: All the plots were done in Matlab. We take the vertical (horizontal) axis to be the imaginary (real) axis and plot the eigenvalues of $M(t)$. The red dots are the eigenvalues of $M(0)$. To show the dynamics of the eigenvalues as a function of $t$, we plot the eigenvalues of $M(t)$ in the complex plane in gray scale, where at $t=0$ they are shown in white (coincide with the red dots) and darken as $t$ increases till their final position at $t=t_{\max }$ shown in black. The eigenvalues of $M(t)$ at any $0 \leq t \leq t_{\max }$ have the same gray scale color. In Matlab we use "hold on;" to show the eigenvalues for all $t$. In the appendix we provide a Matlab code that can be used to plot the trajectory of the eigenvalues similar to what is done here.

Demo 1: In (Fig. 1. left) we show the spectral dynamics of the Hatano-Nelson model $M(t)=H+\delta t P$, where $M(0) \equiv H$ is 

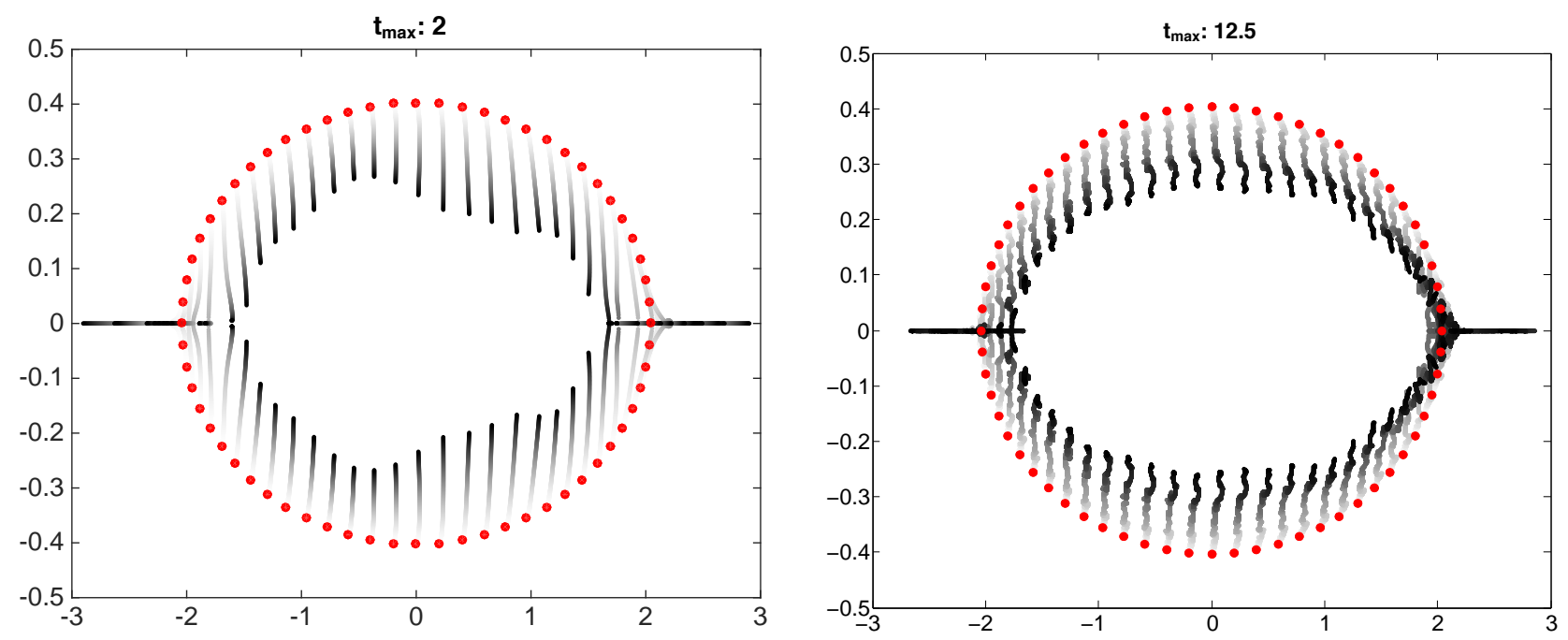

Figure 1: Hatano-Nelson model with $g=0.2$. Left (Demo 1): Small perturbation. Right (Demo 2): Stochastic dynamics. In Demo 2 the final time is much larger because the random impulses imparted at times $0.25 i$ make for much slower net motion over time.

$$
H=\left[\begin{array}{ccccc}
0 & e^{g} & & & e^{-g} \\
e^{-g} & 0 & e^{g} & & \\
& e^{-g} & 0 & \ddots & \\
& & \ddots & \ddots & e^{g} \\
e^{g} & & & e^{-g} & 0
\end{array}\right],
$$

where $g$ governs the non-Hermiticity and $P$ is a real diagonal matrix of random Gaussians. In this figure we took $t_{\max }=2, g=0.2, n=64$, which makes $\|H\|_{2}=2.04$. The boundary terms, i.e., $(1, n)$ and $(n, 1)$ entries, are crucial for the spectral properties [1, Sec. 36].

Comment: When $g \rightarrow 0$, this model coincides with the Anderson model of localization with periodic boundary conditions [16]. However, the latter is a Hermitian model whose properties are quite different from the HatanoNelson model with $g \neq 0$.

Demo 2: In (Fig. 11. right) We took $g=0.2$ as before and take the time discretization $t_{i}=0.25 i$ for $i=0,1, \cdots, 50$, when at each $t_{i}$ an independent matrix $P\left(t_{i}\right)$ is introduced according to $P\left(t_{i}\right)=\operatorname{diag}\left(\epsilon_{1}, \epsilon_{2}, \cdots, \epsilon_{n}\right)$ where $\epsilon_{j}{ }^{\prime}$ s are drawn independently from a standard normal distribution and each $P\left(t_{i}\right)$ is normalized to have a unit $2-$ norm; i.e, $\operatorname{diag}\left[P\left(t_{i}\right)\right]$ is uniform on the unit sphere. A piece-wise linear discrete stochastic process is therefore constructed. We plot the eigenvalues of $M(0.25 i+\delta t)=M(0.25 i)+\delta t P\left(t_{i}\right)$ with $0 \leq \delta t \leq 0.25$ and time steps $\Delta t=0.01$. In (Fig. 1. right), we show the evolution of $M(t)$ for the total time $t \in[0,12.5]$. Note that the eigenvalues move towards the real line as before but make less progress because of the stochastic kicks at times $0.25 i$. Below we show that there is an attraction between complex conjugate pairs that largely governs this behavior of the spectral dynamics in this case. See Section IV] for further theoretical discussion.

Comment: The procedure just described does not provide a smooth stochastic process as it is not differentiable at times $0.25 i$ (a set of measure zero). This issue persists for piece-wise linear discretization where in each interval a new random $P\left(t_{i}\right)$ is used. In fact a continuous Brownian motion provides an example of a continuous map that, with probability one, is nowhere differentiable [5].

\section{Summary of the main results}

In this paper we consider the general problem of the interaction of the eigenvalues of a matrix, $M(t)$, whose entries vary with respect to a real parameter $t$, which we think of as time. Although, eigenvalue repulsion is quite a general feature of Hermitian matrices, attraction of eigenvalues is rarely considered. Below we will first define Eigenvalue 
Attraction (see Def. 2) and give the general equations of the motion of any one of the eigenvalues, which depends on the other eigenvalues and the inertia of the matrix itself. We introduce a new notation more akin to formulations of interacting many-body systems. Theorem 1 gives a simple proof of complex conjugate attraction. Namely, any non-real eigenvalue of $M(t) \in \mathbb{R}^{n \times n}$ attracts its complex conjugate according to

$$
\text { Force of } \overline{\lambda_{i}} \text { on } \lambda_{i}=-i \frac{\left|\mathbf{u}_{i}^{T} \dot{M}(t) \mathbf{v}_{i}\right|^{2}}{\operatorname{Im}\left(\lambda_{i}(t)\right)},
$$

where $\mathbf{v}_{i}$ and $\mathbf{u}_{i}$ are the right (i.e., standard) and left eigenvectors corresponding to the eigenvalue $\lambda_{i}$ and $\overline{\lambda_{i}}$ denotes the complex conjugate of $\lambda_{i}$.

We will apply this to various $M(t)$ such as the Hatano-Nelson model or a convex combination of two deterministic matrices. We then introduce randomness to obtain probabilistic statements. In particular, we consider the pencil of matrices $M(t) \equiv M+\delta t P$, where $M$ is a fixed matrix and $P$ is a random real matrix whose entries have zero mean, finite fourth moments and are independently and identically distributed (iid). This special case of perturbing a fixed matrix comes up often in applications. In this limit and in order to quantify the dominance of the complex conjugate attraction, we calculate the expectation and variance of all other forces excluding the complex conjugate. We prove that when $M$ is a normal matrix (i.e., unitary diagonalizable), the total expected force on any eigenvalue is only due to its complex conjugate, and when $M$ is circulant and $P$ is diagonal, the force only depends on the eigenvalues and that the strength of interaction is the power spectrum of the diagonal entries of $P$, which can be a constant independent of the eigenpairs. For example, if $p_{i i} \sim \mathcal{N}(0,1)$ then the strength of interaction is the power spectrum of white noise. We will prove other results applicable to general circulant matrices and apply them to the Hatano-Nelson model to analyze its spectral dynamics.

We then make a time discretization $0<t_{1}<t_{2}<\cdots$ and define $M(t)$ to be a stochastic process defined by $M\left(t_{i}+\delta t\right)=M\left(t_{i}\right)+\delta t P\left(t_{i}\right)$, where $\delta t \in\left[0, t_{i+1}-t_{i}\right), M(0)$ is a fixed real matrix and each $P\left(t_{i}\right)$ is a real and random, whose entries are independent with zero mean and finite fourth moments. We construct a smooth family

of stochastic processes $\dot{M}_{\epsilon}(t)=P_{\epsilon}(t)$, where $M(t)=\lim _{\epsilon \rightarrow 0} M_{\epsilon}(t)$ and explicitly write down the differential equations governing the motion of any eigenvalue. The expected force of attraction is always

$$
\begin{aligned}
\mathbb{E}\left[\text { Force of } \overline{\lambda_{i}} \text { on } \lambda_{i}\right] & =-i \frac{\sum_{m, \ell} \mathbb{E}\left[p_{m \ell}^{2}\right]\left|u_{i}^{*, m}\right|^{2}\left|v_{i}^{\prime \ell}\right|^{2}}{2 \operatorname{Im}\left(\lambda_{i}\right)} \\
& \stackrel{\text { iid }}{=}-i \frac{\mathbb{E}\left[p^{2}\right]\left\|\mathbf{u}_{\mathbf{i}}\right\|_{2}^{2}}{2 \operatorname{Im}\left(\lambda_{i}\right)}
\end{aligned}
$$

where $\overline{\lambda_{i}}$ is an eigenvalue that is complex conjugate to $\lambda_{i}, \mathbf{v}_{\mathbf{i}}$ and $\mathbf{u}_{\mathbf{i}}^{*}$ are the corresponding right and left eigenvectors respectively and the second equality assumes $\mathbb{E}\left[p_{m, \ell}^{2}\right]$ is the same for all $m$ and $\ell$. Clearly, the attraction is strongest near the real line. Since the proportionality constant depends on the $2-$ norm of the left eigenvector, the force of attraction can be quite strong for ill-conditioned eigenvalues. When this attractive force is dominant over the force exerted by the rest of the eigenvalues, the complex conjugate pair approach one another and eventually collide and "scatter" near and ultimately reside at different points on the real line. At this point, the well-known repulsion mechanism takes over and the reality of the matrix ensures that each eigenvalue remains real.

The motion on the real line is not permanent. In most cases, an eigenvalue that moves about on the real line gets close enough to (i.e., collides with) another eigenvalue on the real line, after which they form a new complex conjugate pair and shoot off into the complex plane. The alternative would be that they would repel and remain on the real line. However, in the majority of cases we investigated, it seems 'energetically' more favorable for them to form a new complex conjugate pair perhaps because there are more degrees of freedom available away from the real line. In this paper, we will not rigorously investigate this to any depth.

As mentioned above, any stochastic process (e.g., Wiener process, Brownian motion) is non-smooth, despite often being continuous. The appearance of a new $P\left(t_{i}\right)$ makes the limits of the derivative from left and right unequal $\dot{M}\left(t_{i}^{>}\right) \neq \dot{M}\left(t_{i}^{<}\right)$, yet there are powerful tools of matrix calculus that can be utilized if $M(t)$ were differentiable. Moreover, from the applied perspective, nothing is instantaneous.

In Section IV, we give the basic definitions of discrete stochastic processes and introduce a smoothing construction that can be used to smoothen any discrete stochastic process with a control over the rapidity of (dis)appearance of 
every $P\left(t_{i}\right)$ within $\left[t_{i}, t_{i+1}\right]$ (see Eq. 34). The original (non-differentiable) stochastic process is

$$
M(t)=\lim _{\epsilon \rightarrow 0} M_{\epsilon}(t)
$$

We will conclude by applying the dynamical perspective developed here to an open problem pertaining to the sparsity of the eigenvalues of random real matrices near the real line, and then list further open problems. It is our hope that this work will prove useful in proving the conjecture stated in Sec $\mathrm{V}$

Remark 2. We make a remark that should otherwise be obvious. In what follows the eigenvalue attraction holds for all $t$ and $M(t)$ does not need to be a perturbation of a fixed matrix. The latter is, however, an application of this work (see Subsection III). Moreover, attraction holds for deterministic evolutions under minimal assumptions and randomness is not a requirement.

\section{EIGENVALUES AS A MANY-BODY SYSTEM}

In this paper we take the point of view that the eigenvalues are interacting identical particles whose motions take place in the complex plane and our goal is to better understand their dynamics. The eigenvalues of $M(t)$ are also functions of time and the $i^{t h}$ eigenvalue is denoted by $\lambda_{i}(t)$. The eigenvalues of a continuously varying $M(t)$ are also continuous in $t$. That is, their motion follows a connected path in the complex plane. This follows from the fact that eigenvalues are roots of a characteristic polynomial, which itself is continuous, and a theorem due to Rouché [17. Chapter 4 ].

Comment: We take the eigenvalues to have unit mass, whereby the acceleration, $\ddot{\lambda}_{i}(t)$, can be identified with the "force" required to produce that acceleration on an eigenvalue. Below we shall use the word force as it provides better intuition.

\section{A. General dynamics of eigenvalues}

Here we follow a derivation similar to that given by T. Tao [2] to obtain the governing dynamical equations for the eigenpairs of a general smoothly varying $M(t)$, although the equations were derived in earlier references. We assume that the eigenvalues are simple. The eigenvalue equations are

$$
\begin{aligned}
M(t) \mathbf{v}_{\mathbf{i}}(t) & =\lambda_{i}(t) \mathbf{v}_{\mathbf{i}}(t) \\
\mathbf{u}_{\mathbf{i}}{ }^{*}(t) M(t) & =\lambda_{i}(t) \mathbf{u}_{\mathbf{i}}{ }^{*}(t),
\end{aligned}
$$

where $\lambda_{i}(t)$ are the eigenvalues, $\mathbf{v}_{\mathbf{i}}(t)$ the (right) eigenvectors, which we take to be normalized, and $\mathbf{u}_{\mathbf{i}}^{*}(t)$ are the left eigenvectors dual to $\mathbf{v}_{\mathbf{i}}(t)$. If we consider the matrix of eigenvectors $\mathbf{V}(t)=\left[\mathbf{v}_{\mathbf{1}}(t) \mathbf{v}_{\mathbf{2}}(t), \ldots, \mathbf{v}_{\mathbf{n}}(t)\right]$, then $\mathbf{u}_{\mathbf{j}}{ }^{*}(t)$ is the $j^{\text {th }}$ row of $\mathbf{V}^{-1}(t)$ and

$$
\mathbf{u}_{\mathbf{j}}^{*}(t) \mathbf{v}_{\mathbf{i}}(t)=\delta_{i j}
$$

Since $\mathbf{v}_{1}(t), \ldots, \mathbf{v}_{n}(t)$ form a basis for $\mathbb{C}^{n}$, any vector $\mathbf{x}$ has the expansion $\mathbf{x}=\sum_{j=1}^{n}\left[\mathbf{u}_{\mathbf{j}}{ }^{*}(t) \mathbf{x}\right] \mathbf{v}_{\mathbf{j}}(t)$. Differentiating Eqs. 4 and 5 with respect to $t$, gives

$$
\begin{aligned}
\dot{M}(t) \mathbf{v}_{\mathbf{i}}(t)+M(t) \dot{\mathbf{v}}_{\mathbf{i}}(t) & =\dot{\lambda}_{i}(t) \mathbf{v}_{\mathbf{i}}(t)+\lambda_{i}(t) \dot{\mathbf{v}}_{\mathbf{i}}(t) \\
\mathbf{u}_{\mathbf{i}}{ }^{*}(t) \dot{M}(t)+\dot{\mathbf{u}}_{\mathbf{i}}{ }^{*}(t) M(t) & =\dot{\lambda}_{i}(t) \mathbf{u}_{\mathbf{i}}^{*}(t)+\lambda_{i}(t) \dot{\mathbf{u}}_{\mathbf{i}}{ }^{*}(t)
\end{aligned}
$$

Multiplying the first equation on the left by $\mathbf{u}_{\mathbf{i}}{ }^{*}(t)$, and using Eq. 5, we obtain the "velocity" of $\lambda(t)$ in the complex plane $^{1}$

$$
\dot{\lambda}_{i}(t)=\mathbf{u}_{\mathbf{i}}^{*}(t) \dot{M}(t) \mathbf{v}_{\mathbf{i}}(t) .
$$

\footnotetext{
${ }^{1}$ We remark that the theory of pseudo-spectra [1] quantifies how far an eigenvalue can wander without quantifying the direction of the motion
} 
In order to compute the acceleration on any eigenvalue we shall need the derivatives of the left and right eigenvectors. They are simple to compute [2],

$$
\begin{aligned}
\dot{\mathbf{v}}_{\mathbf{i}}(t) & =\sum_{j \neq i} \frac{\mathbf{u}_{\mathbf{j}}^{*}(t) \dot{M}(t) \mathbf{v}_{\mathbf{i}}(t)}{\lambda_{i}(t)-\lambda_{j}(t)} \mathbf{v}_{\mathbf{j}}(t)+\eta_{i}(t) \mathbf{v}_{\mathbf{i}}(t) \\
\dot{\mathbf{u}}_{\mathbf{i}}^{*}(t) & =\sum_{j \neq i} \frac{\mathbf{u}_{\mathbf{i}}^{*}(t) \dot{M}(t) \mathbf{v}_{\mathbf{j}}(t)}{\lambda_{i}(t)-\lambda_{j}(t)} \mathbf{u}_{\mathbf{j}}^{*}(t)-\eta_{i}(t) \mathbf{u}_{\mathbf{i}}^{*}(t)
\end{aligned}
$$

where $\eta_{i}(t)$ is a scalar function because a constant multiple of an eigenvector is also an eigenvector. The second derivative, or acceleration, of the eigenvalue $\lambda_{i}(t)$ is obtained by differentiating Eq. 7 one more time,

$$
\ddot{\lambda}_{i}(t)=\dot{\mathbf{u}}_{\mathbf{i}}^{*}(t) \dot{M}(t) \mathbf{v}_{\mathbf{i}}(t)+\mathbf{u}_{\mathbf{i}}{ }^{*}(t) \ddot{M}(t) \mathbf{v}_{\mathbf{i}}(t)+\mathbf{u}_{\mathbf{i}}{ }^{*}(t) \dot{M}(t) \dot{\mathbf{v}_{\mathbf{i}}}(t) .
$$

Using Eqs. 8 and 9, the second derivative becomes [2]

$$
\begin{aligned}
\ddot{\lambda}_{i}(t) & =\mathbf{u}_{\mathbf{i}}{ }^{*}(t) \ddot{M}(t) \mathbf{v}_{\mathbf{i}}(t)+2 \sum_{j \neq i} \frac{\left[\mathbf{u}_{\mathbf{i}}^{*}(t) \dot{M}(t) \mathbf{v}_{\mathbf{j}}(t)\right]\left[\mathbf{u}_{\mathbf{j}}^{*}(t) \dot{M}(t) \mathbf{v}_{\mathbf{i}}(t)\right]}{\lambda_{i}(t)-\lambda_{j}(t)} \\
& =\mathbf{v}_{\mathbf{i}}{ }^{*}(t) \ddot{M}(t) \mathbf{v}_{\mathbf{i}}(t)+2 \sum_{j \neq i} \frac{\left|\mathbf{v}_{\mathbf{i}}^{*}(t) \dot{M}(t) \mathbf{v}_{\mathbf{j}}(t)\right|^{2}}{\lambda_{i}(t)-\lambda_{j}(t)} \text { if the matrix is Hermitian, }
\end{aligned}
$$

where for normal matrices (e.g., Hermitian) one has $\mathbf{u}_{i}=\mathbf{v}_{i}$. As pointed out by Tao, the first term can be seen as the inertial force of the matrix and the second the force of interaction of the eigenvalues. The origin of instantaneous repulsive force between eigenvalues of a Hermitian matrix is easily seen by the second term in Eq. 11] [2]. For example, if $\lambda_{i}(t)>\lambda_{j}(t)$, then the force is positive, the effect of $\lambda_{j}(t)$ in the sum is to push $\lambda_{i}(t)$ to the right. Similarly, if $\lambda_{i}(t)<\lambda_{j}(t)$, the effect of $\lambda_{j}(t)$ is to exert a negative force on $\lambda_{i}(t)$. Moreover the strength of the force is inversely proportional to their distance $\left(1 /\left|\lambda_{i}(t)-\lambda_{j}(t)\right|\right)$ which is clearly strongest when the eigenvalues are closest [2]. The repulsion is at work for Hermitian matrices for all $t$.

Comment: Eqs. (7 111$)$ are essentially the standard first and second order perturbation theory results. See for example, Dirac [18, Section 43] ${ }^{2}$, Kato [19], Wilkinson's wonderful exposition [20] and more recently [21].

\section{B. Definition and Proof of Eigenvalue Attraction}

Below we denote the complex conjugate of an eigenvalue or entry-wise complex conjugation of an eigenvector with an over-line. To better visualize the kinematics of the eigenvalues in the complex plane, we write

$$
\begin{aligned}
& \frac{1}{\lambda_{i}(t)-\lambda_{j}(t)}=\frac{\overline{\lambda_{i}(t)}-\overline{\lambda_{j}(t)}}{\left|\lambda_{i}(t)-\lambda_{j}(t)\right|^{2}} \equiv \frac{\hat{\mathbf{r}}_{i j}}{\left|\mathbf{r}_{i j}\right|} \\
& \mathbf{r}_{i j} \equiv \overline{\lambda_{i}(t)}-\overline{\lambda_{j}(t)} \\
& \hat{\mathbf{r}}_{i j} \equiv \overline{\lambda_{i}(t)}-\overline{\lambda_{j}(t)} \\
&\left|\lambda_{i}(t)-\lambda_{j}(t)\right|
\end{aligned}
$$

\footnotetext{
2 Strictly speaking Dirac's derivation of Equation 10 in Section 43 of this reference, does not hold in general (e.g., non-Hermitian) as the left eigenvectors are not 'bras' in his notation. The latter is a Hermitian conjugate of a standard (right) eigenvector. In his book, Dirac cites (Born, Heisenberg and Jordan, z.f. Physik 35, 565 (1925)) for these formulas
} 
where $\mathbf{r}_{i j}$ is a vector in the complex plane stretching from $\overline{\lambda_{j}(t)}$ to $\overline{\lambda_{i}(t)}$ and $\hat{\mathbf{r}}_{i j}$ is the corresponding unit vector. One could further simplify the notation by denoting the complex number

$$
c_{i j} \equiv \mathbf{u}_{\mathbf{i}}^{*}(t) \dot{M}(t) \mathbf{v}_{\mathbf{j}}(t)
$$

whereby $\dot{\lambda}_{i}(t)=c_{i i}$. In this paper, we denote complex conjugation of the entries of an eigenvector by an over-bar on the corresponding index. For example, $c_{\bar{i}}{ }_{j}=\mathbf{u}_{\mathbf{i}}^{\mathbf{T}}(t) \dot{M}(t) \mathbf{v}_{\mathbf{j}}(t)$ and since $\dot{M}(t)$ is real, $c_{i} c_{\bar{j}} c_{j}=$ $\left|\mathbf{u}_{\mathbf{i}}^{\mathbf{T}}(t) \dot{M}(t) \mathbf{v}_{\mathbf{j}}(t)\right|^{2}$ is a real non-negative number.

With this notation Eqs. 10 and 11 read

$$
\begin{aligned}
& \ddot{\lambda}_{i}(t)=\mathbf{u}_{\mathbf{i}}{ }^{*}(t) \ddot{M}(t) \mathbf{v}_{\mathbf{i}}(t)+2 \sum_{j \neq i} c_{i j} c_{j i} \frac{\hat{\mathbf{r}}_{i j}}{\left|\mathbf{r}_{i j}\right|} \\
& \ddot{\lambda}_{i}(t)=\mathbf{v}_{\mathbf{i}}^{*}(t) \ddot{M}(t) \mathbf{v}_{\mathbf{i}}(t)+2 \sum_{j \neq i}\left|c_{i j}\right|^{2} \frac{\hat{\mathbf{r}}_{i j}}{\left|\mathbf{r}_{i j}\right|} \text { if the matrix is Hermitian. }
\end{aligned}
$$

We think of Eq. 13 as

$$
\begin{aligned}
\ddot{\lambda}_{i}(t) & =\mathbf{u}_{\mathbf{i}}{ }^{*}(t) \ddot{M}(t) \mathbf{v}_{\mathbf{i}}(t)+2 \sum_{j \neq i} c_{i j} c_{j i} \frac{\hat{\mathbf{r}}_{i j}}{\left|\mathbf{r}_{i j}\right|} \\
& =\{\text { Inertial force of } M\}+\sum_{j \neq i}\left\{\text { force of } \lambda_{j} \text { on } \lambda_{i}\right\} .
\end{aligned}
$$

Definition 1. The force between $\lambda_{i}(t)$ and $\lambda_{j}(t)$ is called central if in Eq. [13, $c_{i j} c_{j i}=$ $f\left(\lambda_{i}(t), \lambda_{j}(t), \ldots\right)\left(\lambda_{i}(t)-\lambda_{j}(t)\right)$, where $f\left(\lambda_{i}(t), \lambda_{j}(t), \ldots\right)$ is a real function.

Comment: As shown above $f\left(\lambda_{i}(t), \lambda_{j}(t), \ldots\right)$ is generally a complex-valued function of $i^{\text {th }}$ and $j^{t h}$ eigenvalues and eigenvectors as well as $\dot{M}(t)$.

Definition 2. (Attraction and Repulsion) We say $\lambda_{j}(t)$ attracts (repels) $\lambda_{i}(t)$ if the force between them is central and $f\left(\lambda_{i}(t), \lambda_{j}(t), \ldots\right)$ is a negative (positive) function.

Remark 3. For general (not self-adjoint) matrices the force between any two eigenvalues is not necessarily repulsive nor attractive. As seen in Eq 13 , the orientation of the force is along the ray $c_{i j} c_{j i} \hat{\mathbf{r}}_{i j}$, rendering generally a noncentral force law between the eigenvalues. This contrasts the purely central (and repulsive) nature of the interaction of the eigenvalues for Hermitian matrices (Eq. 14). However, it is generally true that the force law between any two eigenvalues is inversely proportional to their distance.

Theorem 1. (Eigenvalue Attraction) Complex conjugate eigenvalues of $M(t)$ attract (see Def. 2) as long as $M(t)$ is real, the pair is not degenerate, and $\mathbf{u}_{\mathbf{i}}^{\mathbf{T}}(t) \dot{M}(t) \mathbf{v}_{\mathbf{i}}(t) \neq 0$.

Proof. The non-real eigenvalues and eigenvectors of a real matrix come in complex conjugate pairs [22, Chapter 24]. In Eq. 13 the interaction of $\overline{\lambda_{i}(t)}$ and $\lambda_{i}(t)$ is given by the term where $j=\bar{i}$ where $\lambda_{i}(t)-\overline{\lambda_{i}(t)}=2 i \operatorname{Im}\left(\lambda_{i}\right)$ and

$$
\frac{2 c_{i \bar{i}} c_{\bar{i} i}}{\lambda_{i}(t)-\overline{\lambda_{i}(t)}}=-i \frac{\left|c_{i \bar{i}}\right|^{2}}{\operatorname{Im}\left(\lambda_{i}(t)\right)} \text {. }
$$

i. $\operatorname{Im}\left(\lambda_{i}\right)>0$, then the right hand side of Eq. 15 is a negative imaginary number: The effect of $\overline{\lambda_{i}}$ on $\lambda_{i}$ at time $t$ is to push $\lambda_{i}$ downwards along the imaginary axis with a magnitude that is inversely proportional to their distance. The constant of proportionality is $\left.\left|c_{i}\right|^{2}\right|^{2}$ the numerator of Eq. 15

ii. $\operatorname{Im}\left(\lambda_{i}\right)<0$, then the right hand side of Eq. 15 is a positive imaginary number: The effect of $\overline{\lambda_{i}}$ on $\lambda_{i}$ at time $t$ is to push $\lambda_{i}$ upward along the imaginary axis with a magnitude that is inversely proportional to their distance with the same constant of proportionality. 
Comment: Let $M$ be a fixed non-symmetric real matrix and define $M(t)=M+t \mathbb{I}$, where $\mathbb{I}$ is the identity matrix. The eigenvalues of $M(t)$ are those of $M$ shifted by $t$. No attraction is expected. Indeed by Eq. $6 . c_{i i}=\mathbf{u}_{\mathbf{i}}^{\mathbf{T}}(t) \mathbf{v}_{\mathbf{i}}(t)=0$. Moreover, from Eq. 7 we have $\dot{\lambda}_{i}(t)=t$, i.e., a net drift in the complex plane of any eigenvalue with velocity $t$.

Remark 4. Proving that complex conjugate eigenvalues attract does not imply that in the long-run all the eigenvalues will necessarily be real. There are three forces that act on any eigenvalue $\lambda_{i}$ :

1. The inertial force due to $\ddot{M}(t)$.

2. The attraction of its complex conjugate

3. The force of the remaining $n-2$ eigenvalues.

The governing equation is accordingly written as

$$
\ddot{\lambda}_{i}(t)=\mathbf{u}_{\mathbf{i}}{ }^{*}(t) \ddot{M}(t) \mathbf{v}_{\mathbf{i}}(t)-i \frac{\left|c_{i-i}\right|^{2}}{\operatorname{Im}\left(\lambda_{i}\right)}+2 \sum_{j \neq\{i, \bar{i}\}} c_{i j} c_{j i} \frac{\hat{\mathbf{r}}_{i j}}{\left|\mathbf{r}_{i j}\right|} .
$$

Since the force law is inversely proportional to the distance of the eigenvalues, the law of interaction needs to be logarithmic i.e., the potential is $V\left(\left|\mathbf{r}_{i j}\right|\right) \propto \log \left|\mathbf{r}_{i j}\right|$. The logarithmic interaction on the plane implies a short-range force, where the main contribution to the total force comes from the eigenvalues that are in the vicinity.

Proposition 1. The real eigenvalues of $M(t) \in \mathbb{R}^{n \times n}$ interact via a central force.

Proof. Suppose $\lambda_{i}$ and $\lambda_{j}$ are two simple real eigenvalues then the corresponding eigenvectors can be taken to be real. Then $c_{i j}$ and $c_{j i}$ are both real and by Definition 1 interact via a central force.

A natural question then is: how dominant is the complex conjugate attraction in determining the motion of any eigenvalue? One cannot give a general answer to this question as the relevance of complex conjugate attraction depends on the particular $M(t)$ and the particular eigenvalue considered. However, in examining Eq. 16, it is clear that complex conjugate pairs closest to the real line attract strongest. We will show that ill-conditioning enhances the complex conjugate attraction as well. This is expected as ill-conditioning generally implies higher sensitivity to perturbations [1, 22]. Below we provide some exact results and demonstrations for certain general sub-manifolds of real matrices and time evolutions that are of interest. Mathematically, whether the second term in Eq. 16 dominates the time evolution depends on its magnitude relative to the first derivative and the other terms on the right hand side (i.e, third term). After some demonstrations of deterministic evolution in the next section, we proceed to calculate the expected values and variances about that expectation for the first and second derivatives when the evolution is driven by randomness. Further, we derive conditions under which the total expected force is only due to the complex conjugate.

Remark 5. When the dominant force is that of the complex conjugate attraction, it is interesting that a matrix with a simple spectrum is forced to form eigenvalue degeneracies even if the perturbation (or stochastic process below) is generic. This happens momentarily when any $\lambda_{i}$ and $\overline{\lambda_{i}}$ collide on the real line, whereby standard perturbation theory and considerations above break down and the colliding eigenvalues generically form $2 \times 2$ Jordan blocks [20. Chapter 1]. Following a result due to Lidskii-Vishik-Lyusternik, each of the degenerate eigenvalues for a small $\delta t$ after gets a correction of order $(\delta t)^{1 / 2}$ with a coefficient determined entirely by specific entries of $\dot{M}(t)$ [23, See for a review]. Consequently and generically these eigenvalues move in different directions.

\section{APPLICATIONS OF EIGENVALUE ATTRACTION}

\section{A. Smooth interpolation between fixed matrices}

As an illustration of Theorem 1. let us take $M(t)=(1-t) M_{1}+t M_{2}$, where $M_{1}$ and $M_{2}$ are two fixed matrices and $t \in[0,1]$; the eigenvalues of $M(t)$ interpolate between the two. In Figs 2 and 3 , as before, the eigenvalues of $M_{1}$ are shown in red filled circles and they darken till their final position, which is the eigenvalues of $M_{2}$ shown in blue diamonds. We take the size of the matrices to be $16 \times 16$ so the trajectories of eigenvalues can be visually traced more easily. We normalize all the matrices to have unit 2-norm. The examples shown in Figs. 2 and 3 are: 

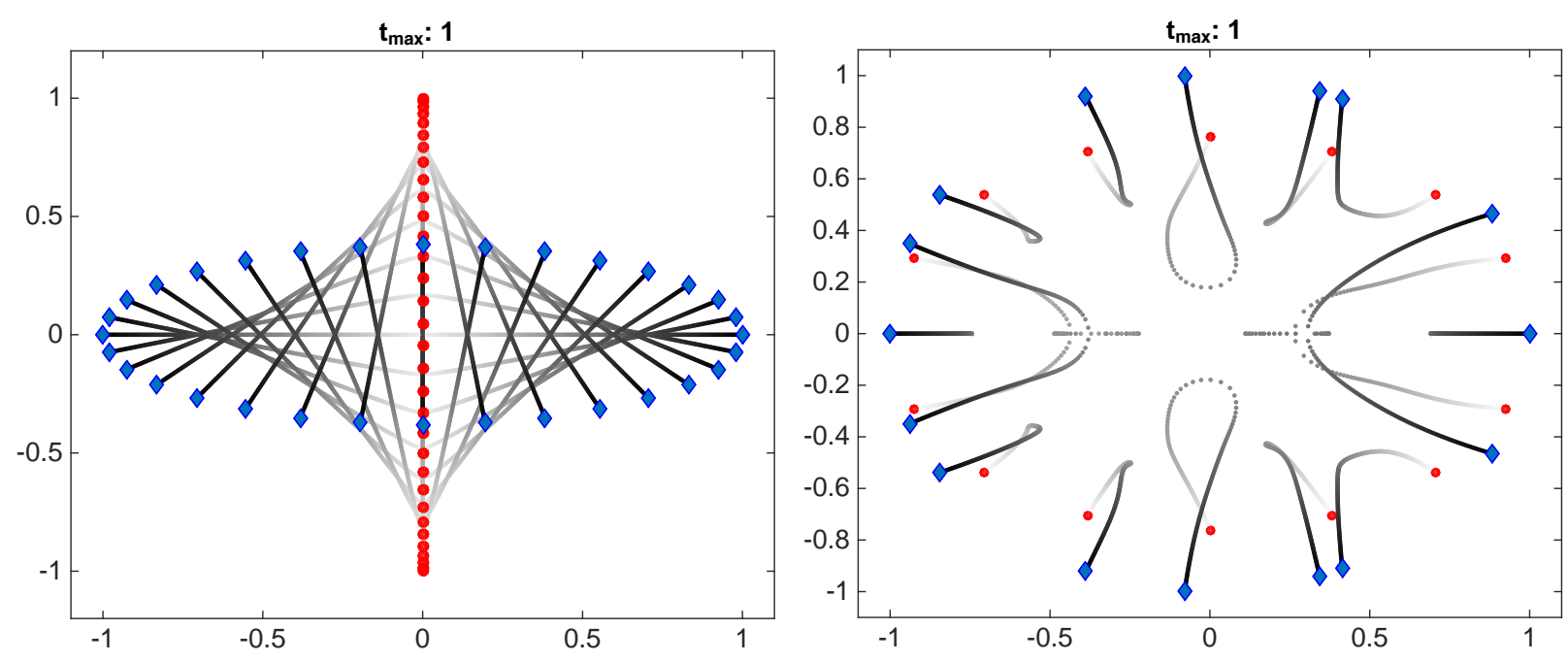

Figure 2: Example 1 (Left): Interpolation between a purely imaginary matrix and the Hatano-Nelson model. Example 2 (Right): Interpolation between the Hatano-Nelson model and a random orthogonal matrix.

Example 1: $M_{1}=\left[\begin{array}{ccccc}0 & +1 & & & \\ -1 & 0 & +1 & & \\ & -1 & 0 & \ddots & \\ & & \ddots & \ddots & +1 \\ & & & -1 & 0\end{array}\right]$ and $M_{2}$ is Hatano-Nelson with $g=-0.4$. Since $M_{1}=-M_{1}^{*}$, its eigenvalues are purely imaginary.

Example 2: $M_{1}$ is Hatano Nelson with $g=1$ and $M_{2}$ is a random orthogonal matrix, hence all the diamonds sit on the unit circle.

Example 3: $M_{1}$ and $M_{2}$ are two real matrices whose entries are iid drawn from a standard normal distribution. That is, $M_{1}$ and $M_{2}$ are drawn from the Ginibre ensemble.

Example 4: $M_{1}$ is Hatano Nelson with $g=-0.3$ and $M_{2}$ is Hatano-Nelson with $g=+0.3$, note that the spectra of $M_{1}$ and $M_{2}$ coincide and that every one of the complex conjugate pairs meet on the real line and walk all the way to the other side.

Example 5: $M_{1}$ and $M_{2}$ are both random orthogonal matrices.

In all the examples shown here, we expect the complex conjugate attraction to be more dominant among other forces for pairs closest to the real line. Note that these complex conjugate pairs first collide and then they move towards their final positions (shown in blue diamonds). A nice corollary is that a real and isolated eigenvalue, will remain real during the time evolution. Since the non-real eigenvalues of $M(t)$ come in complex conjugate pairs, a real eigenvalue cannot move off the real axis. Leaving the real axis can only happen if two eigenvalues collide on the real line and scatter off into the complex plane and remain mirror images (i.e., complex conjugate). For example such collisions are seen in Examples 2 and 3, where in the latter, one can see a cascade of this. That is a complex conjugate pair become real and while moving on the real line a new collision takes the eigenvalues off the real line for a short while, but attraction pulls them back in again for a second collision.

\section{B. Random perturbations of a fixed matrix}

In this section we focus on perturbations of a fixed matrix which come up often in sciences and engineering, where $M(t)$ has the form

$$
M(t)=M+\delta t P
$$

where $M$ and $P$ are $n \times n$ real matrices, $M$ is fixed, $P$ is a random matrix whose entries are independently and identically distributed (iid) with zero mean and finite fourth moments, and $\delta t$ is a small real parameter such that $|\delta t|\|P\| \ll\|M\| ;$ i.e., $\delta t P$ is a perturbation to $M$. The discussion of this section is important for the formulation 

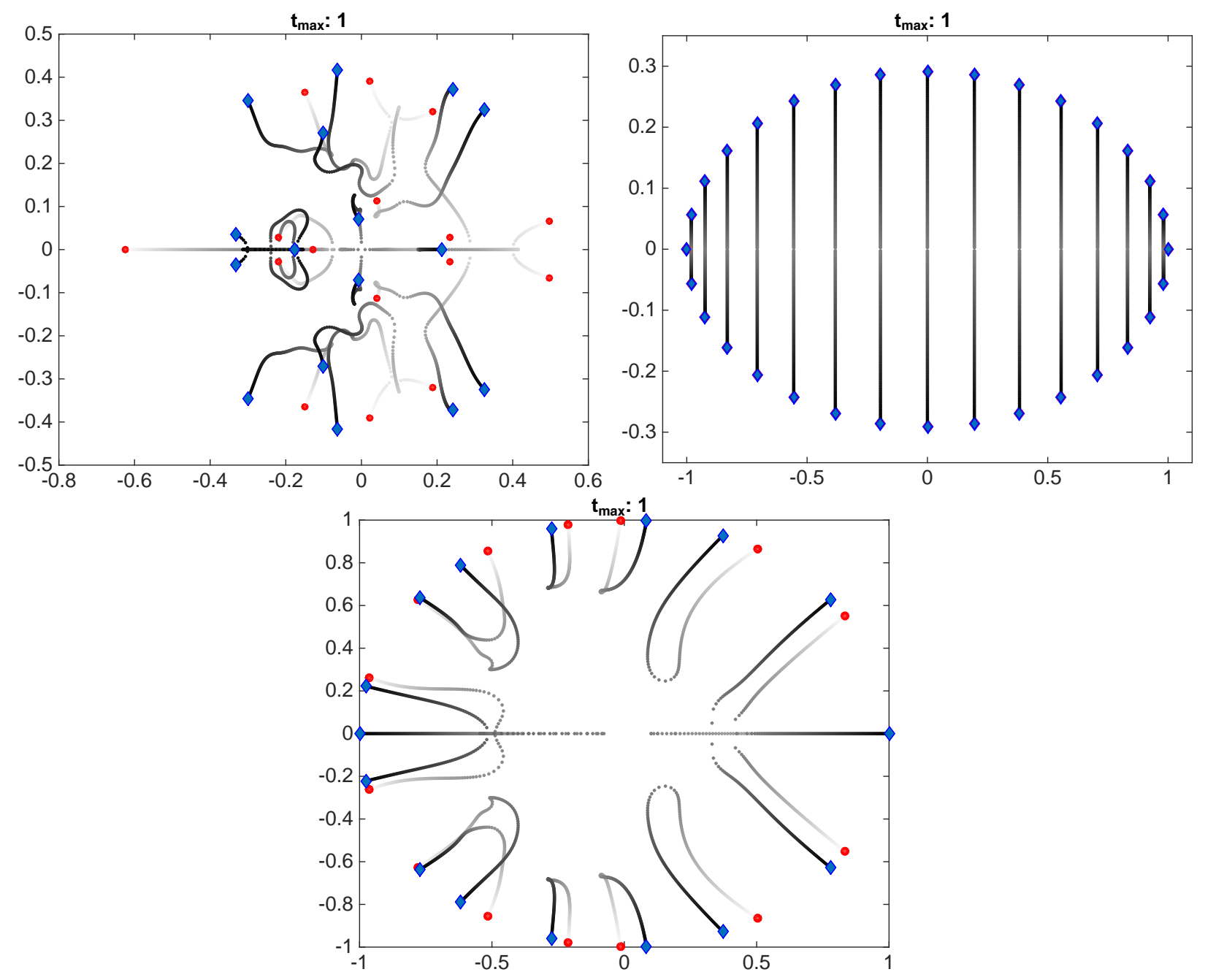

Figure 3: Example 3 (Left): $M_{1}$ and $M_{2}$ are drawn from the Ginibre ensemble. Example 4 (Right): $M_{1}$ and $M_{2}$ are the HatanoNelson model with $g=-0.3$ and $g=+0.3$ respectively. Example 5 (Bottom): $M_{1}$ and $M_{2}$ are two independent random orthogonal matrices.

and analysis of stochastic dynamics of the eigenvalues in Section IV Recall that the velocity and acceleration of an eigenvalue are

$$
\begin{aligned}
& \dot{\lambda}_{i}(t)=c_{i i} . \\
& \ddot{\lambda_{i}}(t)=-i \frac{\left|c_{i-i}\right|^{2}}{\operatorname{Im}\left(\lambda_{i}\right)}+2 \sum_{j \neq\{i, \bar{i}\}} c_{i j} c_{j i} \frac{\hat{\mathbf{r}}_{i j}}{\left|\mathbf{r}_{i j}\right|}
\end{aligned}
$$

where $c_{i j} \equiv \mathbf{u}_{\mathbf{i}}^{*}(t) P \mathbf{v}_{\mathbf{j}}(t)$ and $\frac{\hat{\mathbf{r}}_{i j}}{\left|\mathbf{r}_{i j}\right|}=\frac{1}{\lambda_{i}-\lambda_{j}}$ as before.

\section{First variation}

The expected value, with respect to entries of $P$, of the first variation is

$$
\mathbb{E}\left[\dot{\lambda}_{i}(t)\right]=\mathbb{E}\left[P_{a b}\right]{\overline{u_{i}}}^{a} v_{i}^{b}=0
$$


where for notational convenience from now on we drop the dependence of the eigenpairs on $t$ and sum over the repeated indices that label the components of eigenvectors. We comment that $\mathbb{E}\left[\dot{\lambda}_{i}(t)\right]=0$ even when entries of $P$ are not identically distributed.

The variance is $\mathbb{E}\left[\left|\dot{\lambda}_{i}\right|^{2}\right]-\left|\mathbb{E}\left[\dot{\lambda}_{i}\right]\right|^{2}$. From above $\left|\dot{\lambda}_{i}\right|^{2}=P_{a b} P_{c d} \bar{u}_{i}^{a} v_{i}^{b} u_{i}^{c} \bar{v}_{i}^{d}$ and $\mathbb{E}\left[\dot{\lambda}_{i}\right]=0$. In general, the variance of the first variation denoted by $\sigma_{i, 1}^{2}$ is $\mathbb{E}\left[\left|\dot{\lambda}_{i}\right|^{2}\right]=\mathbb{E}\left[p^{2}\right] \bar{u}_{i}^{a} v_{i}^{b} u_{i}^{a} \bar{v}_{i}^{b}=\mathbb{E}\left[p^{2}\right]\left\|\mathbf{u}_{i}\right\|_{2}^{2}$, where $p$ denotes any entry of the matrix $P$ whose entries are iid. Moreover, if $M$ is a normal matrix then $\left\|\mathbf{u}_{i}\right\|_{2}^{2}=\left\|\mathbf{v}_{i}\right\|_{2}^{2}=1$.

Next suppose that $P=\operatorname{diag}\left(p_{1}, p_{2}, \ldots, p_{n}\right)$ with $\mathbb{E}\left[p_{i}\right]=0$. Then $\left|\dot{\lambda}_{i}\right|^{2}=p_{a} p_{b} \bar{u}_{i}^{a} v_{i}^{a} u_{i}^{b} \bar{v}_{i}^{b}$. Since $\mathbf{u}_{i}^{*} \mathbf{v}_{i}=1$, for diagonal perturbation we have $\mathbb{E}\left[\left|\dot{\lambda}_{i}\right|^{2}\right]=\mathbb{E}\left[p^{2}\right]$.

The special cases are worth summarizing

\begin{tabular}{|c|c|c|}
\hline$M$ & $P$ & $\sigma_{i, 1}^{2}$ \\
\hline General & General & $\mathbb{E}\left[p^{2}\right]\left\|\mathbf{u}_{i}\right\|_{2}^{2}$ \\
\hline General & Diagonal & $\mathbb{E}\left[p^{2}\right]$ \\
\hline Normal & General & $\mathbb{E}\left[p^{2}\right]$ \\
\hline
\end{tabular}

Now suppose that $M$ is a circulant matrix [24] and $P$ is diagonal. In the case of circulant matrices $\mathbf{v}_{j}^{T}=$ $\frac{1}{\sqrt{n}}\left[1, \omega_{j}, \omega_{j}^{2}, \ldots, \omega_{j}^{n-1}\right]$ with $\omega_{j}=\exp (2 \pi i j / n)$. In this case (note that below no expectation is taken)

$$
\dot{\lambda}_{i}=\bar{v}_{i}^{a} p_{a} v_{i}^{a}=p_{a}\left|v_{i}^{a}\right|^{2}=\frac{1}{n} \sum_{a} p_{a} . \quad \text { if the matrix is Circulant. }
$$

This expression is the empirical mean of the diagonal entries of $P$, which for large $n$ tends to zero. Therefore, the dynamics of the eigenvalues are primarily governed by the second variation.

\section{Second variation}

When $M(t)$ is the pencil of matrices given by Eq. $17 \ddot{M}(t)=0, \dot{M}(t)=P$ and

$$
\ddot{\lambda_{i}}=-i \frac{\left|\mathbf{u}_{\mathbf{i}}^{\mathbf{T}} P \mathbf{v}_{\mathbf{i}}\right|^{2}}{2 \operatorname{Im}\left(\lambda_{i}\right)}+\sum_{j \neq\{i, \bar{i}\}} \frac{\left[\mathbf{u}_{\mathbf{i}}^{*} P \mathbf{v}_{\mathbf{j}}\right]\left[\mathbf{u}_{\mathbf{j}}^{*} P \mathbf{v}_{\mathbf{i}}\right]}{\lambda_{i}-\lambda_{j}} .
$$

To quantify the dominance of the attractive force, we calculate the expected value and variance of forces on $\lambda_{i}$ excluding $\bar{\lambda}_{i}$. The general form of the expected force of the remaining $n-2$ eigenvalues on $\lambda_{i}$ (excluding its complex conjugate); i.e. the sum in the foregoing equation or the sum in Eq. 16 is

$$
\begin{aligned}
\mathbb{E} \sum_{j \neq\{i, \bar{i}\}} \frac{\left[\mathbf{u}_{\mathbf{i}}^{*} P \mathbf{v}_{\mathbf{j}}\right]\left[\mathbf{u}_{\mathbf{j}}^{*} P \mathbf{v}_{\mathbf{i}}\right]}{\lambda_{i}-\lambda_{j}} & =\mathbb{E} \sum_{j \neq\{i, \bar{i}\}} \frac{\left[\bar{u}_{i}^{a} p_{a b} v_{j}^{b}\right]\left[\bar{u}_{j}^{c} p_{c d} v_{i}^{d}\right]}{\lambda_{i}-\lambda_{j}}=\sum_{j \neq\{i, \bar{i}\}} \frac{\mathbb{E}\left[p_{a b} p_{c d}\right]\left[\bar{u}_{i}^{a} v_{j}^{b} \bar{u}_{j}^{c} v_{i}^{d}\right]}{\lambda_{i}-\lambda_{j}} \\
& =\mathbb{E}\left[p^{2}\right] \sum_{j \neq\{i, \bar{i}\}} \frac{\left(\mathbf{v}_{\mathbf{i}}^{\mathbf{T}} \mathbf{v}_{\mathbf{j}}\right)\left(\mathbf{u}_{\mathbf{i}}^{*} \overline{\mathbf{u}_{\mathbf{j}}}\right)}{\lambda_{i}-\lambda_{j}},
\end{aligned}
$$

where $p$ denotes any entry of $P$ and $\mathbb{E}\left[p_{a b} p_{c d}\right]=\mathbb{E}\left[p^{2}\right] \delta_{a c} \delta_{b d}$. Although, the attraction is always present, we remark that similar treatment of the first term in Eq. 19 leads to

$$
\mathbb{E}\left[\ddot{\lambda}_{i}\right]=-i \frac{\mathbb{E}\left[p^{2}\right]\left\|\mathbf{u}_{\mathbf{i}}\right\|_{2}^{2}}{\operatorname{Im}\left(\lambda_{i}\right)}+\mathbb{E}\left[p^{2}\right] \sum_{j \neq\{i, \bar{i}\}} \frac{\left(\mathbf{v}_{\mathbf{i}}^{\mathbf{T}} \mathbf{v}_{\mathbf{j}}\right)\left(\mathbf{u}_{\mathbf{i}}^{*} \overline{\mathbf{u}_{\mathbf{j}}}\right)}{\lambda_{i}-\lambda_{j}}
$$




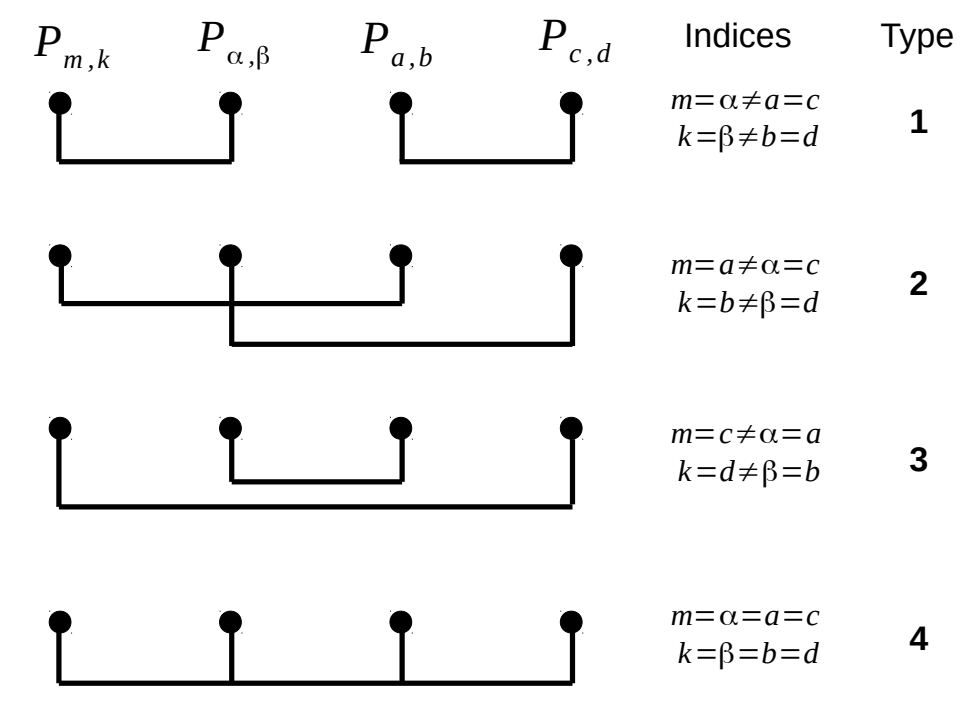

Figure 4: Non-zero contribution in $\mathbb{E}\left\{P_{m k} P_{\alpha \beta} P_{a b} P_{c d}\right\}$

We now calculate the variance of the second term on the right hand side of Eq. 21 , denoted by $\sigma_{i, 2}^{2}$, in Eq. 19

$$
\begin{aligned}
\mathbb{E}\left[\sigma_{i, 2}^{2}\right] & =\mathbb{E}\left\{\left|\sum_{j \neq\{i, \bar{i}\}} \frac{\left[\mathbf{u}_{\mathbf{i}}^{*} P \mathbf{v}_{\mathbf{j}}\right]\left[\mathbf{u}_{\mathbf{j}}^{*} P \mathbf{v}_{\mathbf{i}}\right]}{\lambda_{i}-\lambda_{j}}\right|^{2}\right\}-\left|\mathbb{E} \sum_{j \neq\{i, \bar{i}\}} \frac{\left.\left[\mathbf{u}_{\mathbf{i}}^{*} P \mathbf{v}_{\mathbf{j}}\right]\left[\mathbf{u}_{\mathbf{j}}^{*} P \mathbf{v}_{\mathbf{i}}\right]\right|^{2}}{\lambda_{i}-\lambda_{j}}\right|^{2} \\
& =\sum_{j, \ell \neq\{i, \bar{i}\}} \frac{\mathbb{E}\left[p_{m k} p_{\alpha \beta} p_{a b} p_{c d}\right] \bar{u}_{i}^{m} v_{j}^{k} \bar{u}_{j}^{\alpha} v_{i}^{\beta} u_{i}^{a} \bar{v}_{\ell}^{b} u_{\ell}^{c} \bar{v}_{i}^{d}}{\left(\lambda_{i}-\lambda_{j}\right)\left(\overline{\lambda_{i}}-\overline{\lambda_{\ell}}\right)}-\mathbb{E}^{2}\left[p^{2}\right]\left|\sum_{j \neq\{i, \bar{i}\}} \frac{\left(\mathbf{v}_{\mathbf{i}}^{\mathbf{T}} \mathbf{v}_{\mathbf{j}}\right)\left(\mathbf{u}_{\mathbf{i}}^{*} \overline{\mathbf{u}_{\mathbf{j}}}\right)}{\lambda_{i}-\lambda_{j}}\right|^{2} .
\end{aligned}
$$

There are potentially four nonzero contribution to the first sum shown in Fig. 4 . We proceed to calculate them one by one.

Type 1 : The contribution of this type to Eq. 22 is

$$
\begin{aligned}
\mathbb{E}^{2}\left[p^{2}\right] \sum_{j, \ell \neq\{i, \bar{i}\}} \frac{\bar{u}_{i}^{m} v_{j}^{k} \bar{u}_{j}^{m} v_{i}^{k} u_{i}^{a} \bar{v}_{\ell}^{b} u_{\ell}^{a} \bar{v}_{i}^{b}}{\left(\lambda_{i}-\lambda_{j}\right)\left(\overline{\lambda_{i}}-\overline{\lambda_{\ell}}\right)} & =\mathbb{E}^{2}\left[p^{2}\right] \sum_{j, \ell \neq\{i, \bar{i}\}} \frac{\left(\mathbf{u}_{i}^{*} \overline{\mathbf{u}}_{j}\right)\left(\mathbf{v}_{j}^{T} \mathbf{v}_{i}\right)\left(\mathbf{u}_{i}^{T} \mathbf{u}_{\ell}\right)\left(\mathbf{v}_{\ell}^{*} \overline{\mathbf{v}}_{i}\right)}{\left(\lambda_{i}-\lambda_{j}\right)\left(\overline{\lambda_{i}}-\overline{\lambda_{\ell}}\right)} \\
& =\mathbb{E}^{2}\left[p^{2}\right]\left|\sum_{\ell \neq\{i, \bar{i}\}} \frac{\left(\mathbf{v}_{\ell}^{T} \mathbf{v}_{i}\right)\left(\mathbf{u}_{i}^{*} \overline{\mathbf{u}}_{\ell}\right)}{\lambda_{i}-\lambda_{\ell}}\right|^{2} .
\end{aligned}
$$

where the sum over $j$ is the complex conjugate of the sum over $\ell$. Note that Type 1 cancels the second term in Eq. 22 . Type 2 : Since $\left\|\mathbf{v}_{i}\right\|_{2}^{2}=1$, the contribution of this type to Eq. 22 is

$$
\mathbb{E}^{2}\left[p^{2}\right] \sum_{j, \ell \neq\{i, \bar{i}\}} \frac{\bar{u}_{i}^{a} v_{j}^{b} \bar{u}_{j}^{c} v_{i}^{d} u_{i}^{a} \bar{v}_{\ell}^{b} u_{\ell}^{c} \bar{v}_{i}^{d}}{\left(\lambda_{i}-\lambda_{j}\right)\left(\overline{\lambda_{i}}-\overline{\lambda_{\ell}}\right)}=\mathbb{E}^{2}\left[p^{2}\right]\left\|\mathbf{u}_{i}\right\|_{2}^{2} \sum_{j, \ell \neq\{i, \bar{i}\}} \frac{\left(\mathbf{v}_{\ell}^{*} \mathbf{v}_{j}\right)\left(\mathbf{u}_{j}^{*} \mathbf{u}_{\ell}\right)}{\left(\lambda_{i}-\lambda_{j}\right)\left(\overline{\lambda_{i}}-\overline{\lambda_{\ell}}\right)}
$$

Type 3 : Similarly to Type 1, the contribution of this type to Eq. 22 is calculated to be 


$$
\mathbb{E}^{2}\left[p^{2}\right] \sum_{j, \ell \neq\{i, \bar{i}\}} \frac{\left(\mathbf{u}_{j}^{*} \mathbf{u}_{i}\right)\left(\mathbf{u}_{i}^{*} \mathbf{u}_{\ell}\right)\left(\mathbf{v}_{\ell}^{*} \mathbf{v}_{i}\right)\left(\mathbf{v}_{i}^{*} \mathbf{v}_{j}\right)}{\left(\lambda_{i}-\lambda_{j}\right)\left(\overline{\lambda_{i}}-\overline{\lambda_{\ell}}\right)}=\mathbb{E}^{2}\left[p^{2}\right]\left|\sum_{\ell \neq\{i, \bar{i}\}} \frac{\left(\mathbf{u}_{\ell}^{*} \mathbf{u}_{i}\right)\left(\mathbf{v}_{i}^{*} \mathbf{v}_{\ell}\right)}{\left(\lambda_{i}-\lambda_{\ell}\right)}\right|^{2} .
$$

Type 4 : The contribution of this type to Eq. 22 is

$$
\mathbb{E}\left[p^{4}\right] \sum_{j, \ell \neq\{i, \bar{i}\}} \frac{\bar{u}_{i}^{a} v_{j}^{b} \bar{u}_{j}^{a} v_{i}^{b} u_{i}^{a} \bar{v}_{\ell}^{b} u_{\ell}^{a} \bar{v}_{i}^{b}}{\left(\lambda_{i}-\lambda_{j}\right)\left(\overline{\lambda_{i}}-\overline{\lambda_{\ell}}\right)}=\mathbb{E}\left[p^{4}\right] \sum_{j, \ell \neq\{i, \bar{i}\}} \frac{\left|u_{i}^{a}\right|^{2} \bar{u}_{j}^{a} u_{\ell}^{a}\left|v_{i}^{b}\right|^{2} v_{j}^{b} \bar{v}_{\ell}^{b}}{\left(\lambda_{i}-\lambda_{j}\right)\left(\overline{\lambda_{i}}-\overline{\lambda_{\ell}}\right)} .
$$

We conclude that Eq. 22 can be written as

$$
\begin{aligned}
\mathbb{E}\left[\sigma_{i, 2}^{2}\right] & =\mathbb{E}^{2}\left[p^{2}\right]\left\{\left\|\mathbf{u}_{i}\right\|_{2}^{2} \sum_{j, \ell \neq\{i, \bar{i}\}} \frac{\left(\mathbf{v}_{\ell}^{*} \mathbf{v}_{j}\right)\left(\mathbf{u}_{j}^{*} \mathbf{u}_{\ell}\right)}{\left(\lambda_{i}-\lambda_{j}\right)\left(\overline{\lambda_{i}}-\overline{\lambda_{\ell}}\right)}+\left|\sum_{\ell \neq\{i, \bar{i}\}} \frac{\left(\mathbf{u}_{\ell}^{*} \mathbf{u}_{i}\right)\left(\mathbf{v}_{i}^{*} \mathbf{v}_{\ell}\right)}{\left(\lambda_{i}-\lambda_{\ell}\right)}\right|^{2}\right\} \\
& +\mathbb{E}\left[p^{4}\right] \sum_{j, \ell \neq\{i, \bar{i}\}} \frac{\left|u_{i}^{a}\right|^{2} \bar{u}_{j}^{a} u_{\ell}^{a}\left|v_{i}^{b}\right|^{2} v_{j}^{b} \bar{v}_{\ell}^{b}}{\left(\lambda_{i}-\lambda_{j}\right)\left(\overline{\lambda_{i}}-\overline{\lambda_{\ell}}\right)} .
\end{aligned}
$$

The derivations of this section are purely theoretical. In practice, actual estimates of Eq. 23 would depend on the particular $M(t)$; i.e., $P$ and the eigenpairs of $M$. There are universality results on the eigenvectors of generic Hermitian matrices such as generalized Wigner matrices [25] but not nearly as much is known for generic matrices. Once an estimate is found, convergence to the expected value of the other forces can be proved using standard techniques such as Markov's inequality. Below we discuss special cases that are of theoretical and applied interest.

\section{Normal and circulant matrices}

Now let us confine to the case where $M$ in Eq. [17]is a normal matrix , i.e., unitary diagonalizable [22].

Corollary 1. Suppose $M$ is a normal matrix and $P$ is a random matrix with entries that are iid and have zero mean. Then the total expected force on any eigenvalue is only due to its complex conjugate.

Proof. If $M$ is normal, its eigenvectors form an orthonormal basis for $\mathbb{C}^{n}$ and $\mathbf{u}_{i}=\mathbf{v}_{i}$ with $\mathbf{v}_{\mathbf{i}}^{*} \mathbf{v}_{\mathbf{j}}=\delta_{i j}$. Eq. 20]gives the expected force on $\lambda_{i}$ by all other eigenvalues excluding its complex conjugate, which now reads

$$
\mathbb{E}\left[p^{2}\right] \sum_{j \neq\{i, i\}} \frac{\left|\mathbf{v}_{\mathbf{i}}^{\mathbf{T}} \mathbf{v}_{\mathbf{j}}\right|^{2}}{\lambda_{i}-\lambda_{j}}=0
$$

since the sum over $j$ excludes $\bar{i}$, by orthonormality of the eigenvectors $\mathbf{v}_{\mathbf{i}}^{\mathbf{T}} \mathbf{v}_{\mathbf{j}}=0$. Hence,

$$
\mathbb{E}\left[\ddot{\lambda}_{i}\right]=-i \frac{\mathbb{E}\left[p^{2}\right]}{\operatorname{Im}\left(\lambda_{i}\right)} \quad \text { if the matrix is Normal . }
$$

The total expected force is only due to the complex conjugate

In this case the variance also takes on a simpler expression. In Eq. 23 the first and third sums vanish because $\ell \neq\{i, \bar{i}\}$ and therefore $\mathbf{v}_{\ell}^{T} \mathbf{v}_{i}=0$ and $\mathbf{v}_{i}^{*} \mathbf{v}_{\ell}=0$. Moreover, in the sum in Eq. 23, the sum over $j$ collapses because $\mathbf{v}_{\ell}^{*} \mathbf{v}_{j}=\delta_{\ell j}$ and $\left\|\mathbf{u}_{i}\right\|_{2}^{2}=1$.

Type 4 can be simplified as follows 


$$
\begin{aligned}
\sum_{j \neq\{i, \bar{i}\}} \sum_{\ell \neq\{i, \bar{i}\}} \frac{\mathbb{E}\left[p_{a b}^{4}\right] \overline{v_{i}} a_{j}^{b} \bar{v}_{j}^{a} v_{i}^{b} \bar{v}_{i}^{a} v_{\ell}^{b} \bar{v}_{\ell}^{a} v_{i}^{b}}{\left(\lambda_{i}-\lambda_{j}\right) \overline{\left(\lambda_{i}-\lambda_{\ell}\right)}} & =\mathbb{E}\left[p^{4}\right] \sum_{j \neq\{i, \bar{i}\}} \sum_{\ell \neq\{i, \bar{i}\}} \frac{\left|v_{i}^{a}\right|^{2}\left|v_{i}^{b}\right|^{2} \bar{v}_{j}^{a} v_{\ell}^{a} v_{j}^{b} \bar{v}_{\ell}^{b}}{\left(\lambda_{i}-\lambda_{j}\right) \overline{\left(\lambda_{i}-\lambda_{\ell}\right)}} \\
& \leq \mathbb{E}\left[p^{4}\right]\left|\sum_{\ell \neq\{i, \bar{i}\}} \frac{1}{\lambda_{i}-\lambda_{\ell}}\right|^{2} .
\end{aligned}
$$

because $\sum_{a b}\left|v_{i}^{a}\right|^{2}\left|v_{i}^{b}\right|^{2} \bar{v}_{j}^{a} v_{\ell}^{a} v_{j}^{b} \bar{v}_{\ell}^{b}=\left.\left.\left|\sum_{a}\right| v_{i}^{a}\right|^{2} \bar{v}_{j}^{a} v_{\ell}^{a}\right|^{2} \leq \sum_{a}\left|\bar{v}_{j}^{a} v_{\ell}^{a}\right|^{2} \leq 1$.

Adding the contribution of the four Types in the case where the matrix is normal, we get

$$
\mathbb{E}\left[\sigma_{i, 2}^{2}\right] \leq \mathbb{E}\left[p^{2}\right] \sum_{\ell \neq\{i, \bar{i}\}} \frac{1}{\left|\lambda_{i}-\lambda_{\ell}\right|^{2}}+\mathbb{E}\left[p^{4}\right]\left|\sum_{\ell \neq\{i, \bar{i}\}} \frac{1}{\lambda_{i}-\lambda_{\ell}}\right|^{2} .
$$

where the inequality comes about from the estimate of the Type 4 terms.

How are we to visualize the force between any two eigenvalues? In general one can consider any eigenvalue, $\lambda_{i}$ as a point in the complex plane with coordinates $\left(\operatorname{Re} \lambda_{i}, i \operatorname{Im} \lambda_{i}\right)$ and the unit vector $\hat{\mathbf{r}}_{i j}$, given by Eq. 12 , as one proportional to $\left(\operatorname{Re}\left(\lambda_{i}-\lambda_{j}\right),-i \operatorname{Im}\left(\lambda_{i}-\lambda_{j}\right)\right)$. Looking at the Eq. 13 , we note that the force exerted on $\lambda_{i}$ from $\lambda_{j}$ is in the direction of $c_{i j} c_{j i} \hat{\mathbf{r}}_{i j}$ (recall that $c_{i j}=\mathbf{u}_{\mathbf{i}}^{*}(t) P \mathbf{v}_{\mathbf{j}}(t)$ ). Even in the case of normal matrices, unlike Hermitian matrices, this force is not in general central (attractive or repulsive).

In many lattice models in physics the perturbations are taken to be diagonal, which model the coupling of an external field to the lattice sites. We now consider another example that is relevant for the application such as the Hatano-Nelson model. Take $P=\operatorname{diag}\left(p_{1}, p_{2}, \ldots, p_{n}\right)$ and let $M$ be a circulant matrix; note that now $P$ does not have to be random. For circulant matrices we get ${ }^{3}$

$$
\begin{aligned}
\ddot{\lambda_{k}} & =2 \sum_{j \neq k} \frac{\left|\mathbf{v}_{\mathbf{k}}^{*} P \mathbf{v}_{\mathbf{j}}\right|^{2}}{\lambda_{k}-\lambda_{j}}=-i \frac{\left|v_{k}^{a} p_{a} v_{k}^{a}\right|^{2}}{\operatorname{Im}\left(\lambda_{k}\right)}+2 \sum_{j \neq\{k, \bar{k}\}} \frac{\left|\bar{v}_{k}^{a} p_{a} v_{j}^{a}\right|^{2}}{\lambda_{k}-\lambda_{j}} \\
& =-i \frac{\frac{1}{n^{2}}\left|\sum_{a=1}^{n} p_{a} \omega_{2 k}^{(a-1)}\right|^{2}}{\operatorname{Im}\left(\lambda_{k}\right)}+2 \sum_{j \neq\{k, \bar{k}\}} \frac{\frac{1}{n^{2}}\left|\sum_{a=1}^{n} p_{a} \omega_{j-k}^{(a-1)}\right|^{2}}{\lambda_{k}-\lambda_{j}},
\end{aligned}
$$

since $P^{T}=P$. The quantity $\frac{1}{n} \sum_{a=0}^{n-1} p_{a} \omega_{j-k}^{a-1}$ is the discrete Fourier transformation of the diagonal elements of $P$.

If we assume that the nonzero entries of $P$ are drawn from a standard normal distribution, Eq. 27 is simply the discrete Fourier transform of white noise and $\frac{1}{n^{2}}\left|\sum_{a=0}^{n-1} p_{a} \omega_{j-k}^{a-1}\right|^{2}$ is the power intensity which is a real positive constant independent of the frequency $j-k$ [26], denoted here by $\kappa^{2}$. So the total force is

$$
\ddot{\lambda_{k}}=\kappa^{2}\left\{\frac{-i}{\operatorname{Im}\left(\lambda_{k}\right)}+\sum_{j \neq\{k, \bar{k}\}} \frac{2}{\lambda_{k}-\lambda_{j}}\right\} .
$$

Remark 6. The foregoing equation and the following arguments can apply to the more general cases. For example, in Eq. 13 if $P$ is taken to be diagonal to ensure reality of the numerator and that the numerators have similar magnitudes the following analysis applies.

Proposition 2. Let $M(t)=M+\delta t P$, where $M$ is circulant and $P$ is diagonal with iid entries with zero mean. Any two distinct eigenvalues of $M(t)$ have an expected attractive (repulsive) central force law of interaction between them if and only if their real (imaginary) parts are equal.

\footnotetext{
${ }^{3}$ We denote the eigenvalue by $\lambda_{k}$ instead of $\lambda_{i}$ above because $i \equiv \sqrt{-1}$ appears more in the following discussion.
} 
Proof. The force between the eigenvalues is given by Eq. 28 By def. 2 two eigenvalues $\lambda_{k}$ and $\lambda_{j}$ have a central force between them if for some $\mu \in \mathbb{R}$,

$$
\frac{1}{\lambda_{k}-\lambda_{j}}=\mu\left(\lambda_{k}-\lambda_{j}\right)
$$

Therefore, we would need the unit vector $\hat{\mathbf{r}}_{k j}=\frac{\overline{\lambda_{k}}-\overline{\lambda_{j}}}{\left|\lambda_{k}-\lambda_{j}\right|}$ to be equal to the unit vector $\pm \frac{\lambda_{k}-\lambda_{j}}{\left|\lambda_{k}-\lambda_{j}\right|}$. Let $\lambda_{k}=a+i b$ and $\lambda_{j}=c+i d$ and by def. 2. the force is central if $(a-c)-i(b-d)= \pm[(a-c)+i(b-d)]$. If we take the negative sign then $a=c$ and $b-d$ is free. If we take the positive sign $b=d$ and $a-c$ is free. Therefore, eigenvalues of $M(t)$ that have the same real (imaginary) part attract (repel). Conversely, if $\lambda_{k}$ and $\lambda_{j}$ have the same real parts then $\overline{\lambda_{k}}-\overline{\lambda_{j}}=-i(b-d)$. If $b>d$ then the force that $\lambda_{k}$ experiences is downwards and if $d>b$, it experiences an upward force. The repulsion for eigenvalues with equal imaginary parts is proved using a similar argument. In particular, eigenvalues of a Hermitian matrix repel and non-real complex conjugate eigenvalues attract.

As a concrete example, let $b$ be small and let $x \equiv a-c$ and $y \equiv d-b$. The contribution of the terms from $j$ and $\bar{j}$ to the sum in Eq. 28 is proportional to

$$
\begin{aligned}
& \operatorname{Re}\left\{\frac{1}{\lambda_{k}-\lambda_{j}}+\frac{1}{\lambda_{k}-\overline{\lambda_{j}}}\right\}=x\left\{\frac{1}{x^{2}+y^{2}}+\frac{1}{x^{2}+y^{2}(1+2 b / y)^{2}}\right\} \\
& \operatorname{Im}\left\{\frac{1}{\lambda_{k}-\lambda_{j}}+\frac{1}{\lambda_{k}-\overline{\lambda_{j}}}\right\}=y\left\{\frac{1}{x^{2}+y^{2}}-\frac{1+2 b / y}{x^{2}+y^{2}(1+2 b / y)^{2}}\right\}
\end{aligned}
$$

Evidently any eigenvalue $\lambda_{j}$ repels $\lambda_{k}$ along the real axis since the sign of the force follows the sign of $x$ (See the right figure in Fig. 6. Let us suppose that $2 b / y \equiv \epsilon \ll 1$ (e.g., force of bulk eigenvalues on a $\lambda_{k}$ near the real line), then Eq. 30 becomes

$$
\operatorname{Im}\left\{\frac{1}{\lambda_{k}-\lambda_{j}}+\frac{1}{\lambda_{k}-\overline{\lambda_{j}}}\right\}=-\frac{y \epsilon}{x^{2}+y^{2}}\left\{1-\frac{2 y^{2}}{x^{2}+y^{2}}\right\}
$$

to interpret this equation for now suppose that the imaginary parts of $\lambda_{k}$ and $\lambda_{j}$ are positive, then $\epsilon, y>0$ and the effect of the imaginary part of the pair $\lambda_{j}, \overline{\lambda_{j}}$ on $\lambda_{k}$ is to compress it towards the real axis as long as $x^{2}>y^{2}$. Moreover the imaginary part of the force, unlike the real part, is $\mathcal{O}(\epsilon)$. An entirely similar argument applies to the case where the imaginary parts of $\lambda_{k}$ and $\lambda_{j}$ are negative. Hence as long as the difference of the real parts is larger than that of the imaginary parts, there is a compressive push towards the real line from any complex conjugate pair of eigenvalues on $\lambda_{k}$ with a small net magnitude. In many examples of circulant matrices the pair of eigenvalues closest to the real line appear on the "edges" of the spectrum and the assumptions made above are applicable (for example see the Hatano-Nelson model below and Fig. 6). We conclude that in such cases, the eigenvalues closest to the real line become real as a result of the compression just discussed.

\section{Hatano-Nelson model}

Let $H(t)=H+\delta t P$, where $H$ is a circulant matrix given by Eq. 1 and $P$ is a real diagonal matrix with iid entries with zero mean. The eigenpairs of $H$ are

$$
\begin{aligned}
\lambda_{k} & =2\{\cosh g \cos (2 \pi k / n)+i \sinh g \sin (2 \pi k / n)\} \\
\mathbf{v}_{k}^{T} & =\frac{1}{\sqrt{n}}\left[1, \omega_{k}, \omega_{k}^{2}, \ldots, \omega_{k}^{n-1}\right],
\end{aligned}
$$

where $\omega_{k}=\exp (2 \pi i k / n)$. See Fig. 5 for the effect of the asymmetry parameter $g$ on the spectrum of $H$.

Previously we showed that $\dot{\lambda}_{i} \approx 0$ for sufficiently large $n$ (Eq. 18). Therefore the dynamics are governed by the acceleration $\ddot{\lambda}_{k}$. 


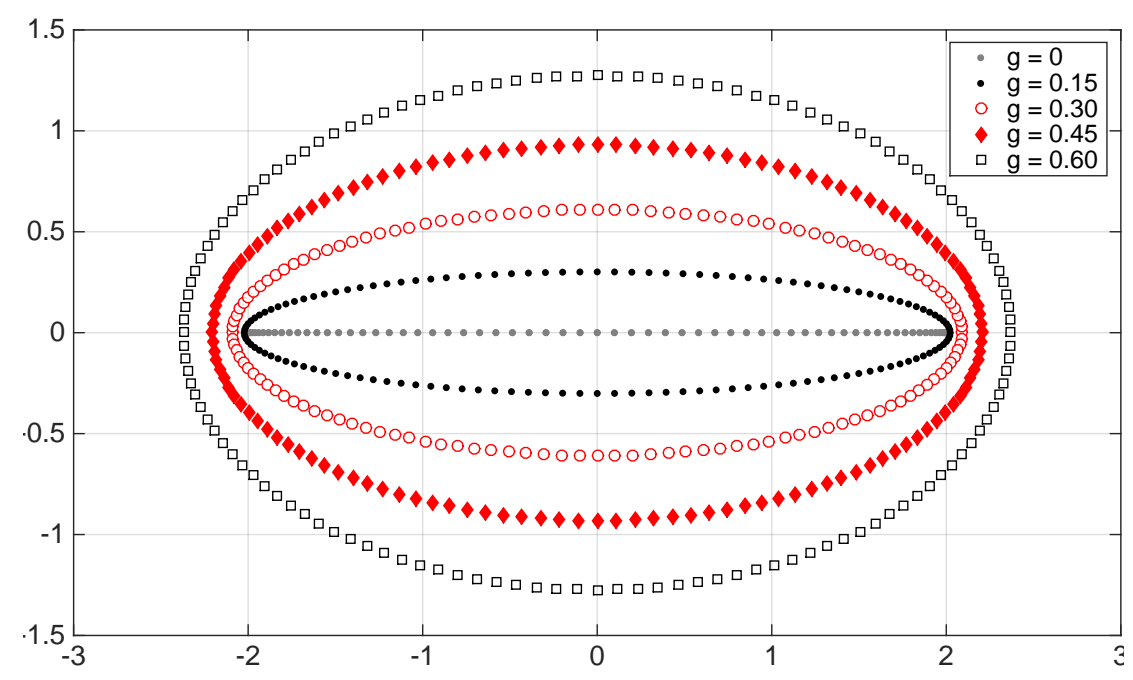

Figure 5: The spectrum of Hatano-Nelson model (Eq. 1) as a function of $g$. When $g$ is small, the spectrum is quite flat for the eigenvalues whose real parts are small.
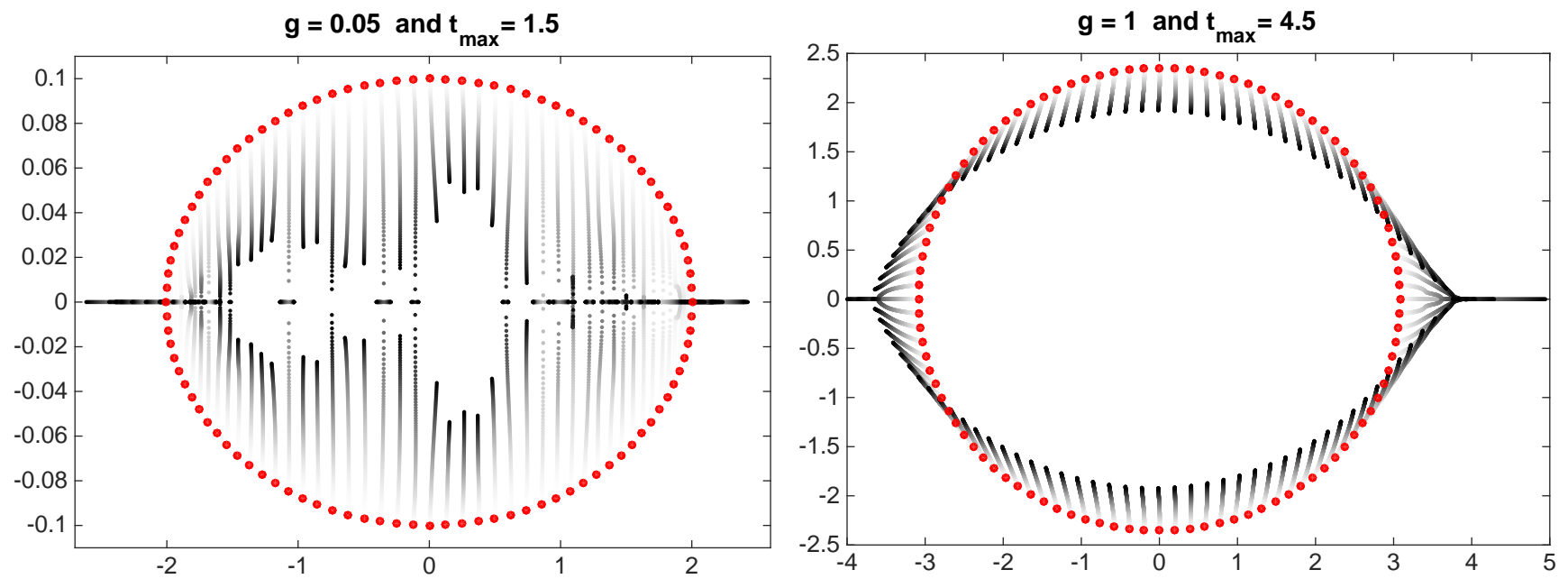

Figure 6: Dynamics of eigenvalues for small (left) $g$ and larger (right) $g$. Also note the difference in range of the imaginary axis and the repulsion along the real axis in agreement with the discussion of the previous section.

Even though $H$ is normal, $H(t)$ is not. However, we observe that for small $g$, the eigenvalues rush to the real line almost undeflected (see the left figure in Fig. 6). Why are the eigenvalues of the Hatano-Nelson model dominated by an attraction towards the real line for relatively large $t$ when $g$ is small?

When $0<g \ll 1, \lambda_{k} \approx 2\{\cos (2 \pi k / n)+i g \sin (2 \pi k / n)\}$ and Eq. 28 becomes

$$
\ddot{\lambda_{k}} \approx \kappa^{2}\left\{\frac{-i}{2 \operatorname{Im}\left(\lambda_{k}\right)}+\sum_{j \neq\{k, \bar{k}\}} \frac{1}{2[\cos (2 \pi k / n)-\cos (2 \pi j / n)]}-i \frac{g[\sin (2 \pi k / n)-\sin (2 \pi j / n)]}{2[\cos (2 \pi k / n)-\cos (2 \pi j / n)]^{2}}\right\}
$$

In the foregoing sum we calculate the contribution of the sum of two terms given by $j$ and $\bar{j}$. Ignoring $\mathcal{O}\left(g^{2}\right)$, we get 


$$
\frac{1}{[\cos (2 \pi k / n)-\cos (2 \pi j / n)]}-i \frac{g \sin (2 \pi k / n)}{[\cos (2 \pi k / n)-\cos (2 \pi j / n)]^{2}} .
$$

Since $\operatorname{Im}\left(\lambda_{k}\right) \approx g \sin (2 \pi k / n)$, the total force is

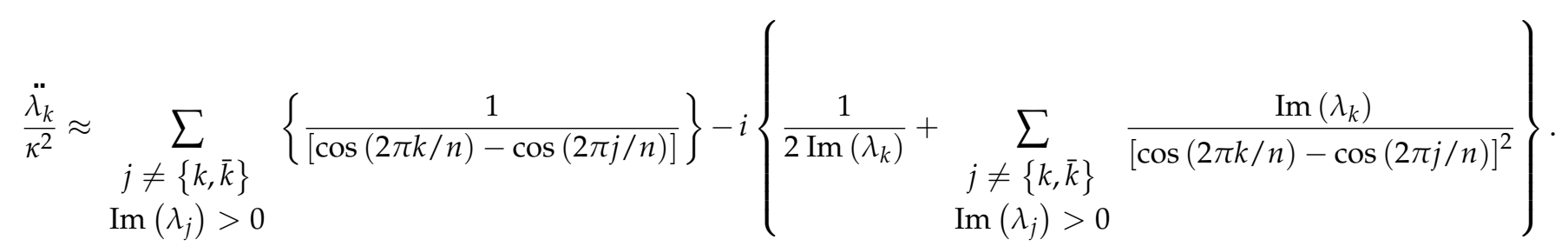

We conclude that there is a net pull of any eigenvalue towards the real line as $\operatorname{Im}\left(\lambda_{k}\right)>0$ gives a force in the $-i$ direction and $\operatorname{Im}\left(\lambda_{k}\right)<0$ gives a force in $+i$ direction. Moreover, the real part of the force of any eigenvalue on $\lambda_{k}$ is repulsive as can easily be seen by analyzing the sign of the denominator of the first sum in the foregoing equation. The eigenvalues rush towards the real line and flow outwards away from the origin (See Fig. 6).

Comment: Take $k=n / 4$, where $\lambda_{n / 4}=i 2 g$, it is easy to see that $\operatorname{Re}\left(\ddot{\lambda}_{\frac{n}{4}}\right)=0$ by rewriting the real part of the $\operatorname{sum}(\mathrm{Eq} \cdot 31$ ) as

$$
\operatorname{Re}\left(\frac{\lambda_{n / 4}^{\ddot{k}}}{\kappa^{2}}\right)=\sum_{\substack{j \neq\{k, \bar{k}\} \\ \operatorname{Im}\left(\lambda_{j}\right)>0}} \frac{-1}{\cos (2 \pi j / n)}=\sum_{\ell=1}^{n / 4-1} \frac{-1}{\cos (2 \pi(n / 4+\ell) / n)}-\frac{1}{\cos (2 \pi(n / 4-\ell) / n)}=0 .
$$

So the net force on this eigenvalue is purely imaginary. In this limit (i.e., $g \ll 1$ ), the spectrum is an ellipse with semi-minor axis $i 2 g$ and semi-major axis 2 (Figs. 5 and 6). The spectrum has small imaginary variations (i.e., is quite flat) for the eigenvalues with small real parts. Therefore the force on these eigenvalues is approximately purely imaginary explaining their almost undeflected rush towards the real line (Fig. 6).

Although $H$ is a circulant matrix and hence normal, $H+\delta t P$ is not, but the deviation from normality is mild for small $g$ as the following shows. The following analysis combined with Corollary 1 . implies that the expected force on any eigenvalue in this model is only due to its complex conjugate even when $\delta t$ is not small. We quantify the degree of non-normality by looking at $\left[H(t), H^{T}(t)\right]=\delta t\left\{[H, P]-\left[H^{T}, P\right]\right\}$, which is calculated to be

$$
\left[H(t), H^{T}(t)\right]=2 \delta t \sinh g\left[\begin{array}{cccccc}
0 & p_{1}-p_{2} & 0 & \cdots & 0 & p_{n}-p_{1} \\
p_{1}-p_{2} & 0 & p_{2}-p_{3} & & & 0 \\
0 & p_{2}-p_{3} & \ddots & \ddots & & \vdots \\
\vdots & & \ddots & 0 & p_{n-2}-p_{n-1} & 0 \\
0 & & & p_{n-2}-p_{n-1} & 0 & p_{n-1}-p_{n} \\
p_{n}-p_{1} & 0 & \ldots & 0 & p_{n-1}-p_{n} & 0
\end{array}\right] .
$$

Let us take each $p_{i}$ to be randomly distributed with mean $\mu$, then the commutator matrix in an expectation sense (with respect to entries) is the zero matrix. For example, if we take $p_{i}$ to be normally distributed with mean $\mu$ and variance $\sigma^{2}$ (i.e., $\mathcal{N}\left(\mu, \sigma^{2}\right)$ ), then $X_{i} \equiv\left(p_{i+1}-p_{i}\right) \sim \mathcal{N}\left(0,2 \sigma^{2}\right)$ and each entry has mean zero and variance $4 t^{2} \sinh ^{2} g$. Therefore fluctuations are small so long as $g$ is small where the variance is approximately $4 t^{2} g^{2}$.

Remark. We intentionally did not use the Frobenius norm to quantify normality as it would not appreciate the entries of the commutator being zero in an expected sense despite $\mathbb{E}(p) \neq 0$. Take as a measure of non-normality the ratio $\left\|\left[H(t), H^{T}(t)\right]\right\|_{F} /\|H\|_{F}^{2}$. But $\|H\|_{F}^{2}=2 n \cosh 2 g$ and $\left\|\left[H(t), H^{T}(t)\right]\right\|_{F}^{2}=2 t \sinh g\left\{2 \sum_{i}\left(p_{i+1}-p_{i}\right)^{2}\right\}$, where $p_{n+1}=p_{1}$. and $\sum_{i} X_{i}^{2} \sim 2 \sigma^{2} \chi_{n-1}^{2}$, where $\chi_{n-1}^{2}$ denotes a chi-square distribution with $n-1$ degrees of freedom and we have 

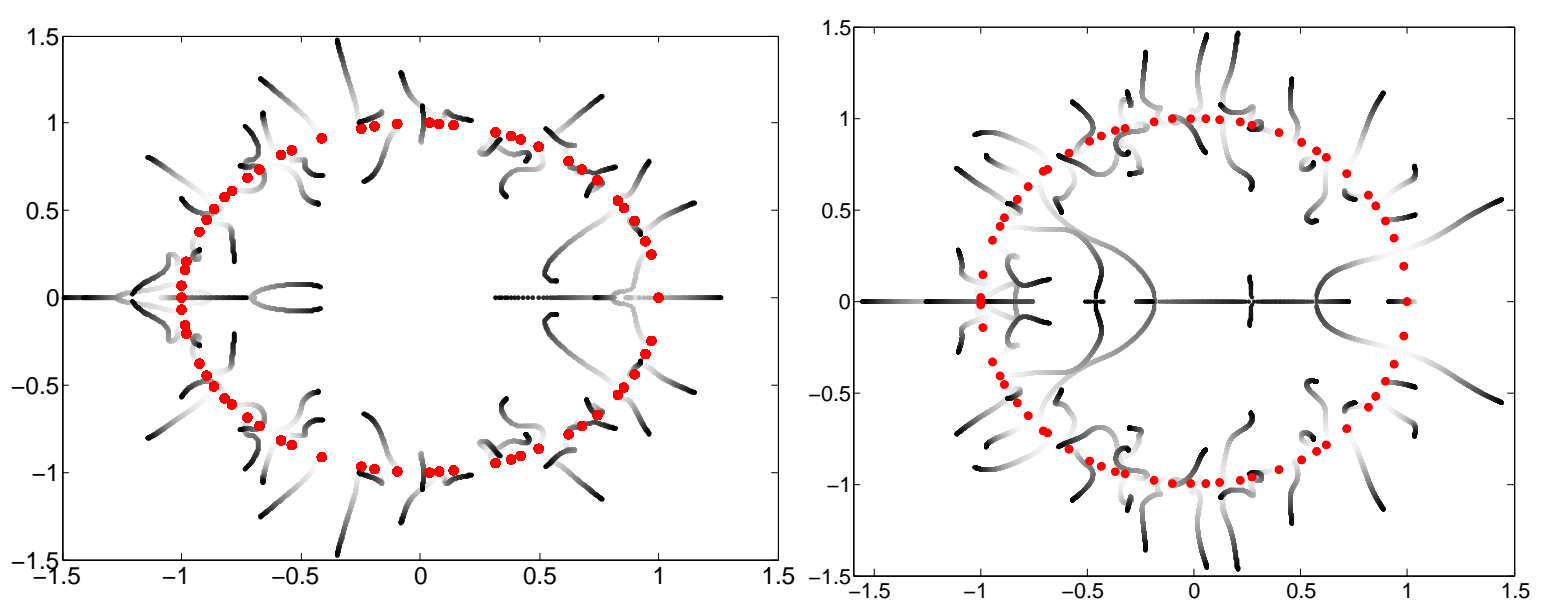

Figure 7: Trajectories of the eigenvalues of $M+\delta t P$. Left: $M$ is an orthogonal matrix and $t_{\max }=2 . P$ is a real random Gaussian matrix with norm 1 . Right: $M$ is an orthogonal matrix and $P$ is a random \pm 1 matrix whose norm is 2 and final time is $t_{m a x}=0.74$.

$$
\frac{\left\|\left[H(t), H^{T}(t)\right]\right\|_{F}}{\|H\|_{F}^{2}} \sim \frac{\sigma \sqrt{2 t \sinh g}}{n \cosh 2 g} \chi_{n-1} .
$$

The mean of the $\chi$-distribution is $\sqrt{2} \Gamma[(n+1) / 2] / \Gamma(n / 2)$, which for large $n$ is approximately $\sqrt{2 n}$ and we have

$$
\mathbb{E}\left[\frac{\left\|\left[H(t), H^{T}(t)\right]\right\|_{F}}{\|H\|_{F}^{2}}\right]=\frac{2 \sigma \sqrt{t \sinh g}}{\sqrt{n} \cosh 2 g} .
$$

Comment: Consider $g \gg 1$, where $H$ becomes proportional to a permutation matrix. In this limit the foregoing expectation is zero. Second take $g \ll 1$, in which case the expectation is approximately $2 \sigma \sqrt{\frac{t g}{n}}$; in this limit the matrix is approximately symmetric.

\section{E. Further illustrations}

Suppose we perturb a $64 \times 64$ real orthogonal matrix, $M$, with $\delta t P$, where $P$ is a real random matrix and $0 \leq \delta t \leq$ $t_{\max }$ with Gaussian entries. In Fig. 7(left) we show the motion of the eigenvalues and indicate $t_{\max }$ for each and on the right we take $P$ to be a random \pm 1 matrix

An application of this work is a better understanding of the origin of real eigenvalues in the Hatano-Nelson model as discussed above (Fig. 1). In Fig. 6 one sees the formation of wings mentioned Sec. If Despite the base case being the same, the motion of the eigenvalues of the Hatano-Nelson model in Figs. 1 (left) and Fig. 6 is much more uniform than in Fig. 1 (right). In the latter plot in the course of the evolution there were 50 intervals with a different random $P$ acting in each. Although continuous, this makes the motion jittery.

In Fig. 8 we take $M$ and $P$ to be two independent $32 \times 32$ real random Gaussian matrices and normalize them to have a unit 2 -norm. Here we also see cases where complex conjugate pairs become real eigenvalues first and then as a result of yet another encounter leave the real line by forming a complex conjugate pair with the newly encountered eigenvalue.

\section{SPECIAL CASE OF STOCHASTIC DYNAMICS OF THE EIGENVALUES}

\section{A. A discrete stochastic process}

Suppose $M(t)$ is a discrete stochastically varying matrix with $M \equiv M(0)$ being a fixed real $n \times n$ matrix. We discretize time $0=t_{0}<t_{1}<\cdots$, and define the evolution of $M(t)$ for any $t_{i} \leq \delta t \leq t_{i+1}$ by a piece-wise linear 


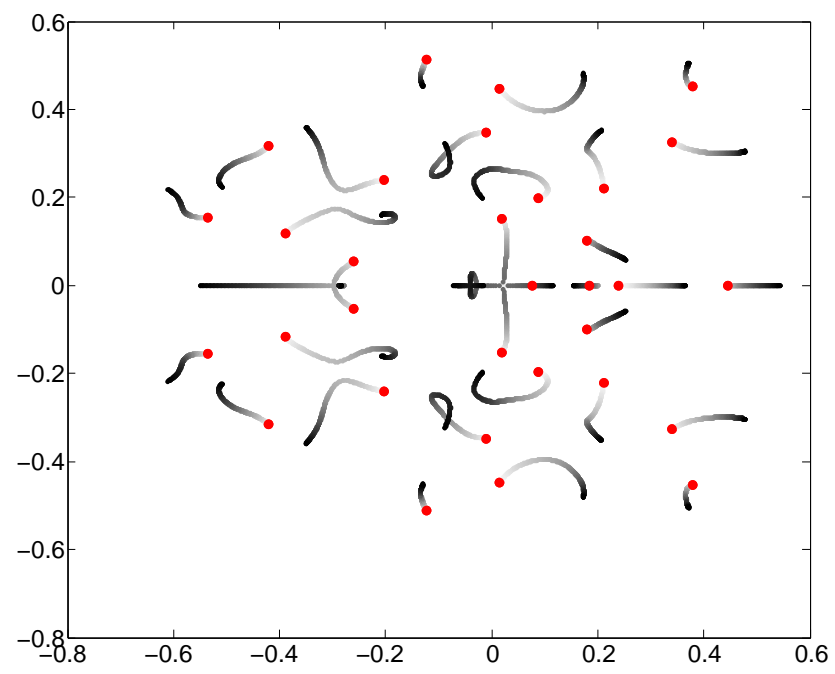

Figure 8: Eigenvalues of $M+\delta t P$, where $M$ is a real Gaussian random matrix of size 32 with unit $2-$ norm. $P$ is a real random Gaussian matrix with unit $2-$ norm. We took $t_{\max }=0.5$.

stochastic process

$$
M\left(t_{i}+\delta t\right)=M\left(t_{i}\right)+\delta t P\left(t_{i}\right)
$$

where $\delta t \in\left[0, t_{i+1}-t_{i}\right)$ and each $P\left(t_{i}\right)$ is a random matrix impulse whose entries are independent and have mean zero i.e., $\mathbb{E}\left[p_{j k}\right]=0 \forall j, k$. The eigenvalues of a continuous stochastic process are continuous in $t$ and within every interval $\left(t_{i}, t_{i+1}\right)$ the results of Subsection III B apply.

Had we used $\sqrt{\delta t}$ in Eq. 32, and defined the process such that $M\left(t_{i}+\delta t\right)-M\left(t_{i}\right)=\sqrt{\delta t} P_{i} \sim N(0, \delta t)_{\mathbb{R}^{n} \times \mathbb{R}^{n}}$, then $M(t)$ would define a discrete Wiener process, which is very special type of a stochastic process. The square root of $\delta t$ is to satisfy the requirement that the variance grows linearly with time. The natural geometry would then be a random walk on the space of $n \times n$ real matrices [5, Chapter 3].

\section{B. Smoothened discrete stochastic process}

In practice nothing develops infinitely fast and no impulse acts instantly. It is more satisfactory to have a controlled smooth, albeit potentially rapidly changing, formulation of the stochastic impulse. To this end, in what follows we define a smooth version of the stochastic impulse, denoted by $P_{\epsilon}(t)$, that in the limit of $\epsilon \rightarrow 0$ becomes Eq. 32 . Let

$$
P_{\epsilon}(t)=\sum_{i \geq 0} P\left(t_{i}\right) W_{\epsilon}\left(t ; t_{i}, t_{i+1}\right),
$$

where each $P\left(t_{i}\right)$ is as before and we define the window function $W_{\epsilon}\left(t ; t_{i}, t_{i+1}\right)$ to be (see Fig. 9 )

$$
W_{\epsilon}\left(t ; t_{i}, t_{i+1}\right)=\left\{\begin{array}{cc}
\Theta\left(t_{i+1}\right)-\Theta\left(t_{i}\right) & t_{i}+\epsilon<t<t_{i+1}-\epsilon \\
B_{-}\left(t ; t_{i}, t_{i+1}\right) & t \leq t_{i}+\epsilon \\
B_{+}\left(t ; t_{i}, t_{i+1}\right) & t \geq t_{i+1}-\epsilon
\end{array}\right.
$$

with $\epsilon<\left(t_{i+1}-t_{i}\right) / 2$, and $\Theta$ being the Heaviside function. $B^{+}$and $B^{-}$are the right and left sections of the modified bump function [27] respectively shown in Fig. 9] such that they reach zero at $t_{i}$ and $t_{i+1}$ and are scaled to have 1 as their maxima (Fig. 9). Mathematically, they are

$$
B_{-}\left(t ; t_{i}, t_{i+1}\right)=\left\{\begin{array}{cc}
e^{1-\frac{1}{1-\left[\left(t-t_{i}-\epsilon\right) / \epsilon\right]^{2}}} & t_{i} \leq t \leq t_{i}+\epsilon \\
0 & \text { otherwise }
\end{array}\right.
$$




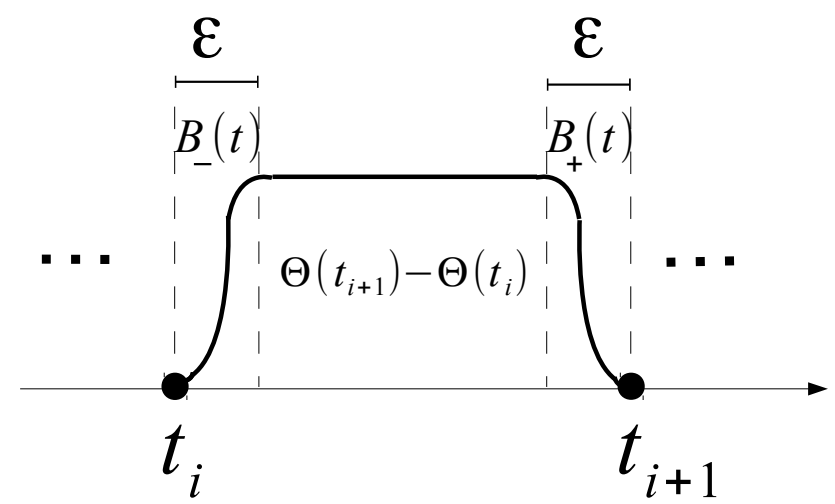

Figure 9: The smooth window function $W_{\epsilon}\left(t ; t_{i}, t_{i+1}\right)$ for $t_{i} \leq t \leq t_{i+1}$.

and

$$
B_{+}\left(t ; t_{i}, t_{i+1}\right)=\left\{\begin{array}{cc}
e^{1-\frac{1}{1-\left[\left(t-t_{i+1}+\epsilon\right) / \epsilon\right]^{2}}} & t_{i+1}-\epsilon \leq t \leq t_{i+1} \\
0 & \text { otherwise }
\end{array}\right.
$$

We think of $B_{ \pm}\left(t ; t_{i}, t_{i+1}\right)$ as equations for the boundary layers near every $t_{i}$. Moreover, the desired independence of time intervals in the discrete stochastic process is guaranteed by the independence of $P\left(t_{i}\right)^{\prime}$ s and their confinement to $t_{i} \leq t \leq t_{i+1}$ by $W_{\epsilon}\left(t ; t_{i}, t_{i+1}\right)$.

It is easy to check that $P_{\epsilon}(t)$ is differentiable everywhere ${ }^{4}$. More specifically, for $\epsilon>0$, it is smooth $\left(C^{\infty}\right)$ everywhere but on a set of measure zero (i.e., all $t_{i}$ ), where it is only $C^{1}$. We shall need the differentiable property below. With these definitions the smooth version of Eq. 32 reads

$$
\dot{M}_{\epsilon}(t)=P_{\epsilon}(t),
$$

with the base case $M \equiv M_{\epsilon}(0)$ being a fixed real $n \times n$ matrix. $M(t)$, which is not differentiable at any $t_{i}$, is recovered by $\lim _{\epsilon \rightarrow 0} M_{\epsilon}(t)$.

Since $P_{\epsilon}(t)$ is random and differentiable, $\lambda(t)$ 's are distinct with probability one. That is $\lambda_{i}(t)$ has an open neighborhood around it that does not contain any other eigenvalue and can be taken to be a smooth function of $t$ [2]. We shall investigate the eigenvalues of $M(t)$.

The smoothing procedure is not necessary if one is only interested in the spectral properties inside a single interval such as in Sec. III E In this case, attraction holds for $t \in\left(t_{i}, t_{i+1}\right)$; i.e., outside the boundary layers.

From our derivations leading to Eq. 34 it now becomes obvious that, for small $\epsilon$, inside the boundary layers, i.e., times $\left(t_{i}-\epsilon, t_{i}+\epsilon\right)$, the dominant force on any eigenvalue is the inertial force $\mathbf{u}_{\mathbf{i}}{ }^{*}(t) \dot{P}_{\epsilon}(t) \mathbf{v}_{\mathbf{i}}(t)$, because

$$
\begin{aligned}
& \mathbf{u}_{\mathbf{i}}{ }^{*}(t) \dot{P}_{\epsilon}(t) \mathbf{v}_{\mathbf{i}}(t) \sim 1 / \epsilon^{2} \quad t_{j}<t<t_{j}+\epsilon \\
& \mathbf{u}_{\mathbf{i}}{ }^{*}(t) \dot{P}_{\epsilon}(t) \mathbf{v}_{\mathbf{i}}(t) \sim-1 / \epsilon^{2} \quad t_{j}-\epsilon<t<t_{j} .
\end{aligned}
$$

For all other times this term is zero and the interaction of the eigenvalues, given by the second term (Eq. 13), governs the force. We will further discuss this and the continuum limit in the next section.

Below to simplify notation, we let $\lambda_{i}(t)=\lambda_{i}, \mathbf{v}_{\mathbf{i}}(t)=\mathbf{v}_{\mathbf{i}}$, and $\mathbf{u}_{\mathbf{i}}(t)=\mathbf{u}_{\mathbf{i}}$, whereby, Eq. 10 reads

\footnotetext{
${ }^{4}$ It is possible to construct $C^{\infty}$ versions of such window functions such as the Planck-taper window function[27. pp. 127-134]; however, $W$ has a simple form with the basic differentiability properties needed here.
} 


$$
\begin{aligned}
\ddot{\lambda}_{i}(t) & =\left(\mathbf{u}_{i}^{*} \dot{P}_{\epsilon}(t) \mathbf{v}_{\mathbf{i}}\right)+2 \sum_{j \neq i} \frac{c_{i j} c_{j i}}{\lambda_{i}-\lambda_{j}} \\
& \doteq(\text { Stochastic Force })+\sum_{j \neq i}\left\{\text { Force of } \lambda_{j} \text { on } \lambda_{i}\right\}
\end{aligned}
$$

where $c_{i j}=\mathbf{u}_{\mathbf{i}}^{*} P_{\epsilon}(t) \mathbf{v}_{\mathbf{j}}$.

Corollary 2. (attraction) Let $M \equiv M_{\epsilon}(0)$ be a real matrix that evolves according to $\dot{M}_{\epsilon}(t)=P_{\epsilon}(t)$, where $P_{\epsilon}(t)$ is given by Eq. 33 Then for all $t$, any complex conjugate pair of eigenvalues of $M_{\epsilon}(t)$ attract (as in Definition 2). Moreover the expected stochastic force is zero.

Proof. The attraction immediately follows from the previous proof of attraction for small perturbations. Let us denote $P_{\epsilon}(t)$ and its components by $P_{\epsilon}$ and $p_{m \ell}$ respectively. The first variation of the eigenvalues in Eq. 7, using index notation reads $\dot{\lambda}_{i}=\mathbf{u}_{i}^{*, m} p_{m \ell} \mathbf{v}_{i}^{\ell}$.

We comment that for small $t_{i} \leq \delta t \leq t_{i+1}, \mathbf{u}_{\mathbf{i}}{ }^{*}$ and $\mathbf{v}_{\mathbf{i}}$ are taken to be eigenvectors of $M\left(t_{i}\right)$ which are independent of $P_{\epsilon}\left(t_{i}+\delta t\right)$. Hence the right-hand sides, in the proof below, are accurate up to $\mathcal{O}(\delta t)$. The first variation is

$$
\mathbb{E}\left[\dot{\lambda}_{i}\right]=\bar{u}_{i}^{m} \mathbb{E}\left[p_{m \ell}\right] v_{i}^{\ell}=0,
$$

since $\mathbb{E}\left[p_{m \ell}\right]=0$ by assumption. From Eq. 10 we have

$$
\mathbb{E}\left[\ddot{\lambda}_{i}\right]=\bar{u}_{i}^{m} \mathbb{E}\left[\left(\dot{P}_{\epsilon}\right)_{m \ell}\right] v_{i}^{\ell}+2 \mathbb{E} \sum_{j \neq i} \frac{\left(\mathbf{u}_{\mathbf{j}}^{*} P_{\epsilon} \mathbf{v}_{\mathbf{i}}\right)\left(\mathbf{u}_{\mathbf{i}}^{*} P_{\epsilon} \mathbf{v}_{\mathbf{j}}\right)}{\lambda_{i}-\lambda_{j}} .
$$

where $\mathbb{E}\left[\left(\dot{P}_{\epsilon}\right)_{m \ell}\right]=\sum_{i} \mathbb{E}\left[p_{m \ell}\left(t_{i}\right)\right] \dot{W}_{\epsilon}\left(t ; t_{i}, t_{i+1}\right)=0$. We have

$$
\mathbb{E}\left[\ddot{\lambda}_{i}\right]=-i \frac{\mathbb{E}\left[p^{2}\right]\left\|\mathbf{u}_{\mathbf{i}}\right\|_{2}^{2}}{\operatorname{Im}\left(\lambda_{i}\right)}+2 \mathbb{E}\left[p^{2}\right] \sum_{j \neq\{i, \bar{i}\}} \frac{\left(\mathbf{v}_{\mathbf{i}}^{\mathbf{T}} \mathbf{v}_{\mathbf{j}}\right)\left(\mathbf{u}_{\mathbf{i}}^{*} \overline{\mathbf{u}}_{\mathbf{j}}\right)}{\lambda_{i}-\lambda_{j}}
$$

Therefore the expected force of attraction between complex conjugate pairs is

$$
\mathbb{E}\left[\text { force of } \overline{\lambda_{i}} \text { on } \lambda_{i}\right]=-i \frac{\mathbb{E}\left[p^{2}\right]\left\|\mathbf{u}_{\mathbf{i}}\right\|_{2}^{2}}{\operatorname{Im}\left(\lambda_{i}\right)}
$$

Remark 7. Complex conjugate eigenvalues and eigenvectors that ultimately become real or those that are initially real and eventually become a complex conjugate pair must first become equal. Since the motion of the eigenvalues is continuous and the matrix is real, the transition from a complex conjugate pair to two real eigenvalues or vice versa requires that they first become equal. This corollary may be obvious but perhaps is interesting in that the degeneracy of eigenvalues is forced under a generic evolution.

Corollary 3. The expected force of attraction of the complex conjugate eigenvalues is directly proportional to the square of the 2-norm of the left eigenvector and the variance of the entries of the perturbation matrix.

This is an immediate consequence of Eq. 40. The numerator in Eq. 40 can change the strength of interaction, most notably because of $\left\|\mathbf{u}_{\mathbf{i}}{ }^{*}\right\|_{2}^{2}$, which for non-normal matrices can become quite large.

Now suppose we want to define a continuous stochastic process where in the equations of motion we first take $\epsilon \rightarrow 0$ and then $\delta t \rightarrow 0$. The first limit will produce two Dirac delta functions at each $t_{i}$ whereby $\lim _{t \rightarrow t_{i}} \dot{P}(t)=$ $-P\left(t_{i-1}\right) \delta\left(t_{i}\right)$ and $\lim _{t \rightarrow t_{i}^{>}} \dot{P}(t)=P\left(t_{i}\right) \delta\left(t_{i}\right)$; therefore the function is not differentiable at $t_{i}$. Lastly, $\delta t \rightarrow 0$ will ensure that the stochastic process is nowhere differentiable as one expects from continuous Brownian motion ideas. So what does this mean for eigenvalue attraction? The infinitesimally close delta function impulses dominate the time evolution (Eqs. 35 and 36) with a zero mean force on any eigenvalue.

Remark 8. For a real stochastic process as before the requirement of $M_{\epsilon}(t)$ being normal for all times demands that $\left[M_{\epsilon}\left(t_{i}\right)-M_{\epsilon}^{T}\left(t_{i}\right), P_{\epsilon}\left(t_{i}\right)\right]=0$ which generally is not met for generic $P_{\epsilon}\left(t_{i}\right)$. 


\section{FURTHER DISCUSSIONS AND OPEN PROBLEMS}

Strongly attracting complex conjugate pairs ultimately coalesce on the real line and scatter like billiard balls and move about on the real line. Thereafter they can act like "normal" eigenvalues and repel. In particular, the inevitability of collision between an eigenvalue and its complex conjugate prevents any eigenvalue from crossing the real line (changing the sign of its imaginary part), without a second encounter.

The motion of the eigenvalues is constrained by the reality of the matrix; the eigenvalue distribution remains symmetric about the real axis. As can be seen in the Figures, an interesting scenario is when two complex conjugate eigenvalues attract and coalesce on the real line, after which they move in opposite directions on the real line till one of them collides with another (real) eigenvalue. Subsequently, the newly encountered eigenvalue and one of the original complex conjugate eigenvalues can momentarily become equal, then move off the real line as a new complex conjugate pair (see Fig. 8, the left figure in Fig. 3 and the right figure in Fig. 7 for examples). At times they simply repel each other and remain real.

We emphasize that the proof of complex conjugate attraction is one of the many forces and at any given instance the net force on any eigenvalue (Eq. 10 and Eq. 37) is the result of the sum of forces of the remaining $n-1$ eigenvalues. In particular, a random collision can take place in the complex plane between eigenvalues that are not complex conjugates and cause a deviation in the path of an eigenvalue that initially moved towards the real line.

It should be clear that the attraction proved in this work does not imply that the long-time behavior is an aggregation of all the eigenvalues on the real line. For a fixed $\delta t$, and over long times, the process loses memory of the initial condition (i.e., $M(t=0)$ ) and ultimately behaves like a random walk on the space of $\mathbb{R}^{n \times n}$ matrices. In fact, Edelman, Kostlan, and Shub [28] showed that for an $n \times n$ real random matrix whose entries are drawn from a standard normal distribution, the expected number of real eigenvalues is approximately $\sqrt{\frac{2 n}{\pi}}$. Later Tao and $\mathrm{Vu}$ [29] proved that matrices whose entries are jointly independent, exponentially decaying, and whose moments match the real Gaussian ensemble to fourth order have $\sqrt{\frac{2 n}{\pi}}+o(\sqrt{n})$ real eigenvalues.

It would be interesting to calculate relaxation times for real deterministic matrices that evolve stochastically and see how long it takes for the matrix to start acting "typical" whereafter the results just mentioned determine the expected behavior.

For any simple eigenvalue $\lambda_{i}$, the condition number [1, p. 474] is a function of the angle between the left and right eigenvectors denoted by $\theta_{0}$

$$
\kappa_{i}=\frac{\left\|\mathbf{u}_{\mathbf{i}}\right\|\left\|\mathbf{v}_{\mathbf{i}}\right\|}{\left|\mathbf{u}_{\mathbf{i}}{ }^{*} \mathbf{v}_{\mathbf{i}}\right|}=\left\|\mathbf{u}_{\mathbf{i}}\right\| \equiv \frac{1}{\left|\cos \theta_{0}^{\ell}\right|},
$$

where we used the orthogonality condition (Eq. 6) and the normality (unit length) of $\mathbf{v}_{i}$. By the Cauchy-Schwarz inequality $\kappa_{i} \geq 1$. An eigenvalue for which $\kappa_{i}=1$ is called a normal eigenvalue and is stable under perturbation, whereas an ill-conditioned eigenvalue has $\kappa_{i} \gg 1$. The right and left eigenvectors associated to an ill-conditioned eigenvalue can become almost orthogonal implying $\kappa_{i} \gg 1$ or equivalently $\left\|\mathbf{u}_{\mathbf{i}}\right\| \gg 1$.

The eigenvalues of normal matrices (e.g., Hermitian, unitary) are very stable under small perturbations. This is not generally the case for non-normal (e.g., Toeplitz) matrices, where small perturbations can change the spectrum significantly [1, 30]. Therefore by Corollary 3 . complex conjugate eigenvalues that are distant; i.e., $\operatorname{Im}\left(\lambda_{i}\right)$ is not necessarily small, can attract strongly if they are ill-conditioned.

\section{A. Does the low density of eigenvalues near the real line result from repulsion?}

It was previously argued that the relatively low density of eigenvalues of real random matrices seen near the real axis results from a repulsion of eigenvalues from the real line [31], [32. Section 6.1](see Fig. 10). Preceding [32], Edelman derived the distribution of the eigenvalues for standard normal random matrices and, interestingly, argued that one might think of the real axis as attracting the nearby eigenvalues [33, Section 2 following Theorem 6.2] (preprint appeared in 1993).

One can conceive of a potentially more complete explanation, where the interaction and dynamics of the eigenvalues take the center stage and not a mysterious interaction with the real axis. To do so, one might need to relate every instance of a real random matrix to the limit of a stochastic process with a base case contained in the deformations of the particular matrix (see below). 

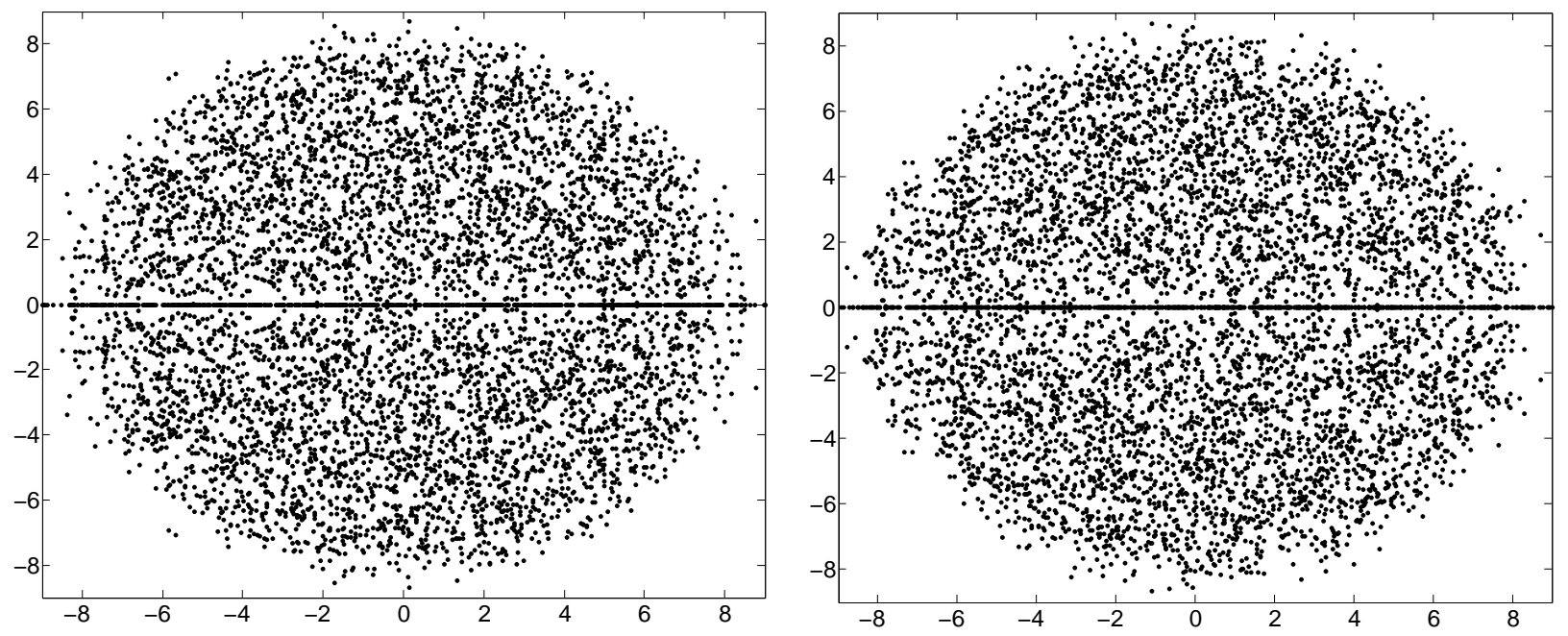

Figure 10: Eigenvalues of 100 instances of $64 \times 64$ real matrices. Left: Entries drawn from a standard normal distribution. Right: Entries are random \pm 1 . Note the aggregation of the eigenvalues on the real axis and their lower nearby density.

In the considerations above, the complex conjugate eigenvalues of a real matrix move more rapidly towards the real line. Moreover, colliding eigenvalues on the real line have a large acceleration when they shoot off the real line. This is easily seen from Eq. 13, real eigenvalues have high accelerations as $\lambda_{i}-\lambda_{j}$ is small and their subsequent motion is either on the real axis or into the complex plane.

In either case when there is an imaginary component to the acceleration, its magnitude is quite high. The former corresponds to high accelerations towards the real line which result in the complex conjugate eigenvalues becoming real. The latter is a strong repulsion away from the real line, shooting the eigenvalues into the complex plane away from the real axis. Therefore, at any given time, on average, one expects a smaller number of eigenvalues to be in the vicinity of the real axis. For large times, when the equilibrium is reached, $M(t)$ will have lost the memory of the initial conditions. At all subsequent times, under the stochastic evolution, some eigenvalues become real (because of attraction) and some move off the real line (because of collisions) and on average about $\mathcal{O}(\sqrt{n})$ of the eigenvalues will be found on the real line.

Large forces between nearby eigenvalues is in no way special to the ones with small imaginary components. However, the reality of the matrix causes an anisotropy- the acceleration of the eigenvalues in the imaginary direction becomes larger.

Conjecture. The low density of eigenvalues of real random matrices near the real axis is the result of the large imaginary components of the acceleration into (attraction of complex conjugate pair) and away from (colliding real eigenvalues) the real axis.

The relative lack of stability of eigenvalues may explain their aggregation on the real line, as well as their low nearby density. In order to settle this conjecture, a first step might be to construct any $n \times n$ real random matrix as a limit of a dynamical process like we did above. In particular, deformations of a given random matrix are also random, so in a way, one can conceive of the stochastic process to be the deformations of a matrix in the neighborhood of the random matrix. Then one can relate the expectation of finding an eigenvalue to the expectation of the time it spends anywhere on the complex plane, which, for real random matrices, would be lower in the vicinity of the real axis.

It is our belief that the repulsion of eigenvalues away from the real line is only part of the story in accounting for their relative low density near the real axis.

\section{B. Further opportunities for future work}

A list of other open problems includes: 
1. What is the probability of collision of eigenvalues on the the real line ${ }^{5}$ The answer to this question would be a helpful step in proving the conjecture above.

2. Estimation of $c_{i j}$ would help quantify the direction and strength of interaction between pairs of eigenvalues.

3. One could give an estimate of the imaginary part of Eq. 31. By doing so one can solve the differential equation to calculate the time it takes for any eigenvalue, $\lambda_{k}$, to reach the real axis, which is the time that $\lambda_{k}$ and $\overline{\lambda_{k}}$ collide and momentarily become degenerate. Indeed, let $u \equiv \operatorname{Im}\left(\lambda_{k}\right)$. The imaginary part of the differential equation (Eq. 31 is of the form $\ddot{u}=f(u)$, which can be solved by first multiplying both sides by $\dot{u}$. That is $\ddot{u} \ddot{u}=\frac{1}{2} \frac{d}{d t}(\dot{u})^{2}=\dot{u} f(u)$ and one has $d(\dot{u})^{2}=2 f(u) d u$, hence $\dot{u}(t)=\sqrt{(\dot{u}(0))^{2}+2 \int f(u) d u}$, which can be integrated once more to solve for $t$ when $u(t)=0$.

4. Study of eigenvector localization, especially as the eigenvectors become more real.

5. Toeplitz matrices provide excellent examples of matrices that can be asymmetric and arise in various applications [1]. It would be interesting to better understand the role of the symbol (e.g. its singularity) [1] in connection with this work.

6. Do new features appear in the operator limit?

7. Application of this work in other areas such as open quantum systems [34, 35], PT-symmetric material [12] and biophysics [13].

\section{Acknowledgements}

I thank Leo P. Kadanoff, Steven G. Johnson, Tony Iarrobino and Gil Strang for discussions and the James Franck Institute at University of Chicago and the Perimeter Institute Canada, for having hosted me over the summer of 2013. I acknowledge the National Science Foundation's support through grant DMS. 1312831.

* Electronic address: q.eigenman@gmail.com

[1] L. N. Trefethen and M. Embree, Spectra and Pseudospectra (Princeton University Press, 2005).

[2] T. Tao, Poincarés legacies: pages from year two of a mathematical blog (American Mathematical Society, 2009).

[3] L. D. Landau and E. M. Lifshitz, Quantum Mechanics (Pergamon Press, 1981).

[4] L. Erdös, B. Schlein, and H.-T. Yau, Int Math Res Notices 3, 436 (2010).

[5] T. Tao, Topics in Random Matrix Theory (Graduate Studies in Mathematics) (American Mathematical Society, March 21, 2012).

[6] F. Dyson, J. Mathematical Phys. 3, 1191 (1962).

[7] N. Hatano and D. R. Nelson, Physical Review B 56, 8651 (1997).

[8] E. Brézin and A. Zee, Nuclear Physics B 509, 599 (1998).

[9] P. Brouwer, P. Silvestrov, and C. Beenakker, Physical Review B 56, 55 (1997).

[10] J. Feinberg and A. Zee, (1997), arXiv:9706218.

[11] H. Widom, Operator Theory: Advances and Applications 71, 1 (1994).

[12] Z. Lin, H. Ramezani, T. Eichelkraut, T. Kottos, H. Cao, and D. N. Christodoulides, Phys. Rev. Lett. 106, 213901 (2011).

[13] D. R. Nelson, Annu. Rev. Biophys. 41, 371 (2012).

[14] I. Y. Goldsheid and B. A. Khoruzhenko, Physical Review Letters 80, 2897 (1998).

[15] J. Bloch, F. Bruckmann, N. Meyer, and S. Schierenberg, Journal of High Energy Physics 8, 1 (2012).

[16] P. W. Anderson, Physical Review 109, 1492 (1958).

[17] G. Stewart and J.-G. Sun, Matrix Perturbation Theory, 1st ed. (Academic Press, 1990).

[18] P. A. M. Dirac, The principles of quantum mechanics, Vol. 4 (Clarendon Press Oxford, 1958).

[19] T. Kato, Perturbation theory for linear operators, Vol. 132 (Springer Science \& Business Media, 1976).

[20] J. H. Wilkinson, The algebraic eigenvalue problem, Vol. 87 (Clarendon Press Oxford, 1965).

[21] T. Tao and V. Vu, Acta mathematica 206, 127 (2011).

[22] L. N. Trefethen and D. B. III, Numerical Linear Algebra (Siam, 1997).

\footnotetext{
${ }^{5}$ This question was posed to us by Freeman Dyson.
} 
[23] J. Moro, J. V. Burke, and M. L. Overton, SIAM Journal on Matrix Analysis and Applications 18, 793 (1997).

[24] R. M. Gray, Toeplitz and Circulant Matrices: A review (Now Pub, 2006).

[25] P. Bourgade and H.-T. Yau, arXiv preprint arXiv:1312.1301 (2013).

[26] P. Stoica and R. L. Moses, Introduction to spectral analysis, Vol. 1 (Prentice hall Upper Saddle River, 1997).

[27] L. W. Tu, An Introduction to Manifold (New York, Springer, 2008).

[28] A. Edelman, E. Kostlan, and M. Shub, J. Amer. Math. Soc. 7, 247 (1994).

[29] T. Tao and V. Vu, (2012), arXiv:1206.1893 [math.PR].

[30] A. Boettcher, M. Embree, and V. I. Sokolov, Math. Comp. 72, 1329 (2003).

[31] A. Mays, Asia Pacific Mathematics Newsletter 4, 8 (2014).

[32] A. Edelman and E. Kostlan, Bulletin (new series) of the American Mathematical Society 32 (1995).

[33] A. Edelman, Journal of Multivariate Analysis 60, 203 (1997).

[34] F. Ticozzi, R. Lucchese, P. Cappellaro, and L. Viola, Automatic Control, IEEE Transactions on 57, 1931 (2012).

[35] F. Ticozzi and L. Viola, Philosophical Transactions of the Royal Society A: Mathematical, Physical and Engineering Sciences 370, 5259 (2012). 


\section{APPENDIX: MATLAB CODE}

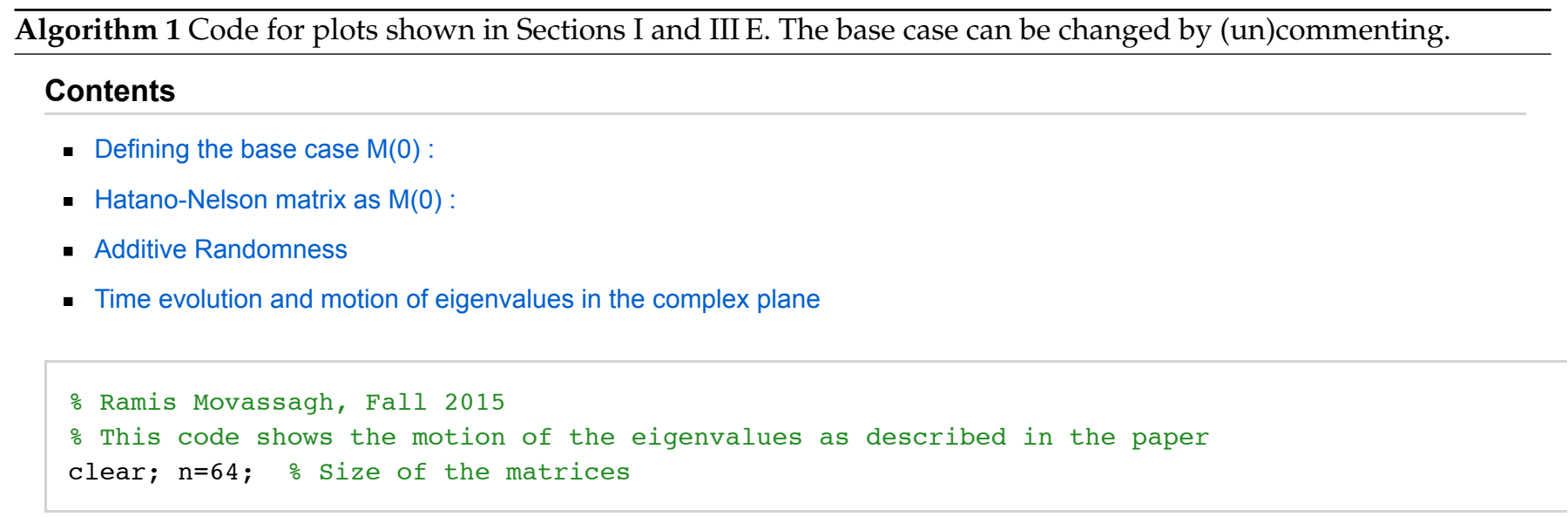

\section{Defining the base case $M(0)$ :}

\% $[\mathrm{H}$ dc $]=\mathrm{qr}(\operatorname{randn}(\mathrm{n}))$; \% random orthogonal

$\% \mathrm{H}=\mathrm{randn}(\mathrm{n})$; \% random Gaussian

\section{Hatano-Nelson matrix as $M(0)$ :}

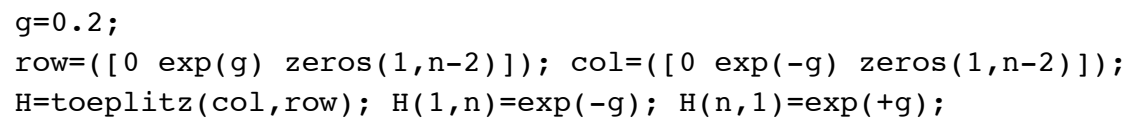

\section{Additive Randomness}

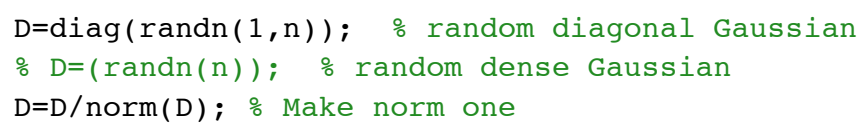

\section{Time evolution and motion of eigenvalues in the complex plane}

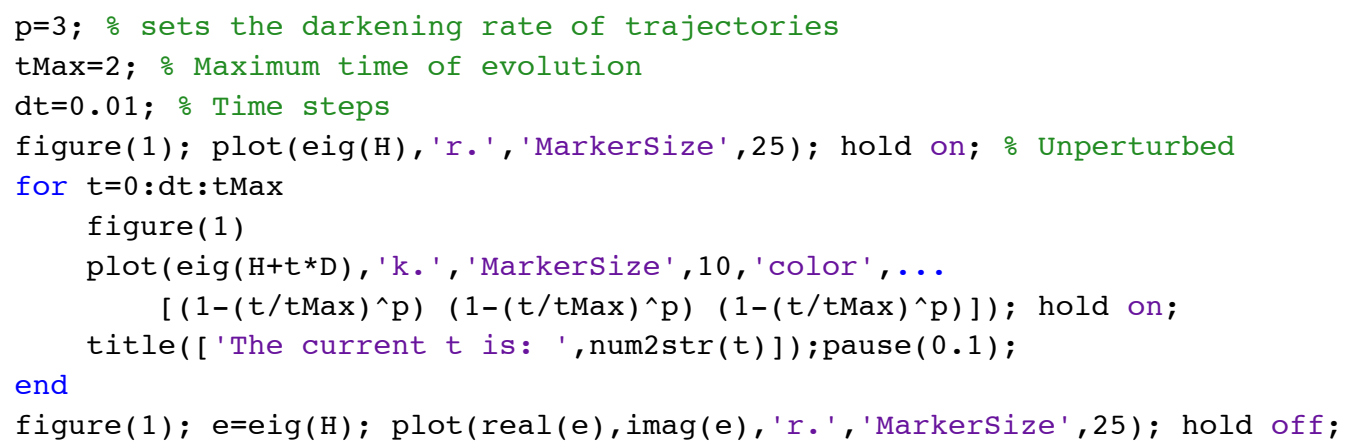

